
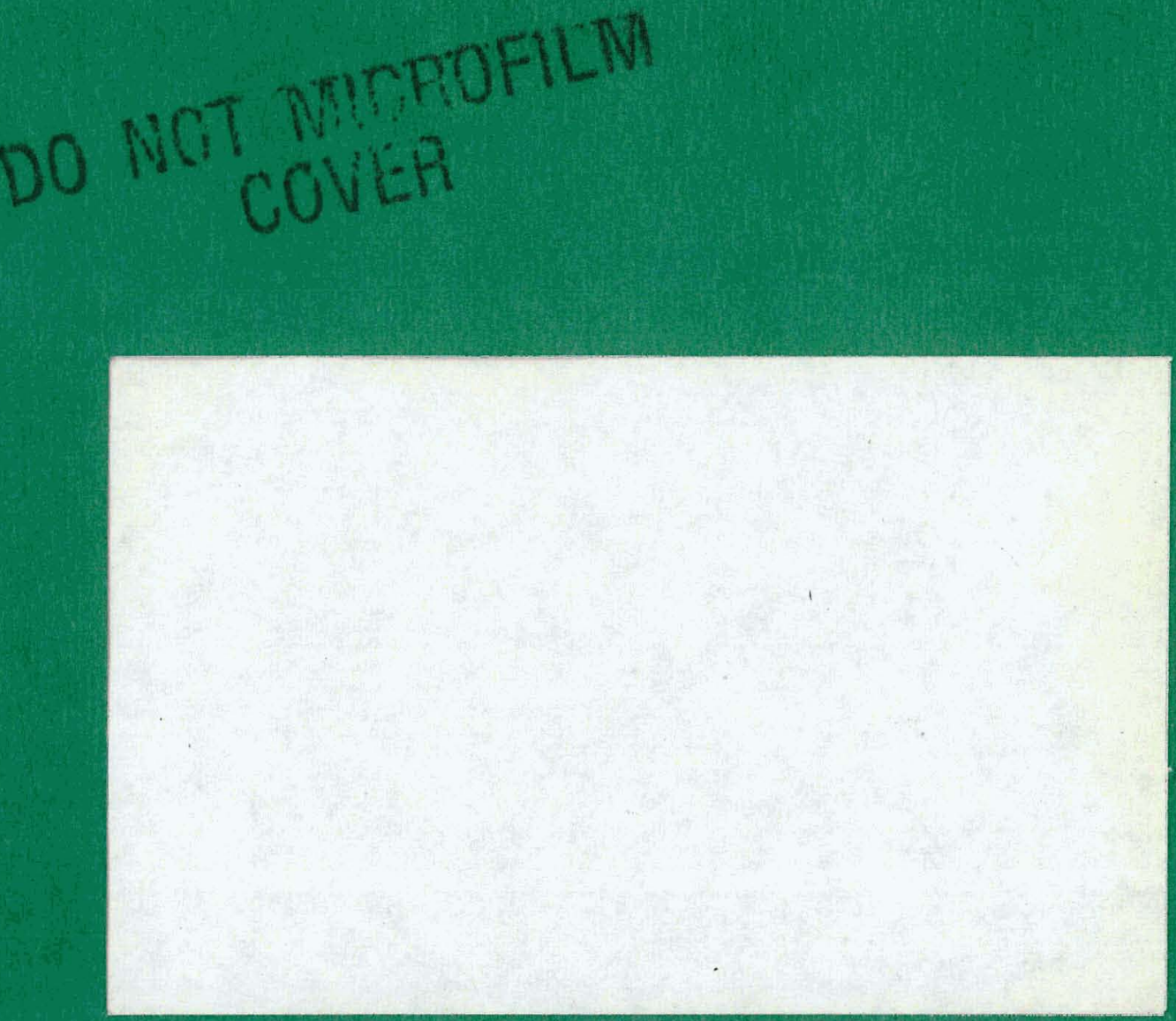

Center for Supercomputing Research \& Development National Center for Supercomputing Applications University of Illinois at Urbana-Champaign 


\section{DISCLAIMER}

This report was prepared as an account of work sponsored by an agency of the United States Government. Neither the United States Government nor any agency Thereof, nor any of their employees, makes any warranty, express or implied, or assumes any legal liability or responsibility for the accuracy, completeness, or usefulness of any information, apparatus, product, or process disclosed, or represents that its use would not infringe privately owned rights. Reference herein to any specific commercial product, process, or service by trade name, trademark, manufacturer, or otherwise does not necessarily constitute or imply its endorsement, recommendation, or favoring by the United States Government or any agency thereof. The views and opinions of authors expressed herein do not necessarily state or reflect those of the United States Government or any agency thereof. 


\section{DISCLAIMER}

Portions of this document may be illegible in electronic image products. Images are produced from the best available original document. 


\title{
AUTOMATIC GENERATION OF SYNCHRONIZATION INSTRUCTIONS FOR PARALLEL PROCESSORS
}

\author{
Samuel Pratt Midkiff
}

May 1986

\section{DISCLAIMER}

This report was prepared as an account of work sponsored by an agency of the United States Government. Neither the United States Government nor any agency thereof, nor any of their employees, makes any warranty, express or implied, or assumes any legal liability or responsibility for the accuracy, completeness, or usefulness of any information, apparatus, product, or process disclosed, or represents that its use would not infringe privately owned rights. Reference herein to any specific commercial product, process, or service by trade name, trademark, manufacturer, or otherwise does not necessarily constitute or imply its endorsement, recommendation, or favoring by the United States Government or any agency thereof. The views and opinions of authors expressed herein do not necessarily state or reflect those of the United States Government or any agency thereof.

Center for Supercomputing Research and Development

University of Illinois

305 . Talbot - 104 South Wright Street

Urhana, IL. 61801-2932

Phone: (217) 333-6223

This work was supported in part by the National Science Foundation under Grant Nos. US NSF DCR84-10110 and US NSF DCR84-06916, the U. S. Department of Energy under Grant No. US DOEDE-FG02-85ER25001, the IBM Donation, and was submitted in partial fulfillment of the requirements for the degree of Master of Science in the Computer Science Department, May 1986. 


\title{
AUTOMATIC GENERATION OF SYNCHRONIZATION INSTRUCTIONS FOR PARALLEL PROCESSORS
}

\author{
BY
}

SAMUEL PRATT MIDKIFF

B.S., University of Kentucky, 1982

\section{THESIS}

Submitted in partial fulfillment of the requirements for the degree of Master of Science in Computcr Scicnce in the Graduale College of the University of Illinois at Urbana-Champaign, 1986

Urbana, Illinois 


\title{
AUTOMATIC GENERATION OF SYNCHRONIZATION INSTRUCTIONS FOR PARALLEL PROCESSORS
}

\author{
Samuel Pratt Midkiff, M.S. \\ Department of Computer Science \\ University of Illinois at Urbana-Champaign, 1986 \\ David Padua, Advisor
}

The development of high speed parallel multi-processors, capable of parallel execution of doacross and forall loops, has stimulated the development of compilers to transform serial FORTRAN programs to parallel forms. One of the duties of such a compiler must be to place synchronization instructions in the parallel version of the program to insure the legal execution order of doacross and forall loops.

This thesis gives strategies usable by a compiler to generate these synchronization instructions. It presents algorithms for reducing the parallelism in FORTRAN programs to match a target architecture, recovering some of the parallelism so discarded, and reducing the number of synchronization instructions that must be added to a FORTRAN program, as well as basic strategies for placing synchronization instructions. These algorithms are developed for two synchronization instruction sets. 


\section{ACKNOWLEDGEMENTS}

Many people have helped and guided me my work on this thesis, and made its completion possible. I would first like to thank David J. Kuck for making it possible for me to work on the Cedar project and serving as my advisor in the first stages of my graduate.career. I would also like to thank Ron Cytron for suggesting this topic to me and offering 'early guidance. David Padua, my current advisor, has been of invaluable help. His ideas and grasp of the issue at hand has always been perceptive, and his suggestions about both form and content have always led to this thesis being better than it might have otherwise. Discussions with him about matters technical and otherwise have always been interesting as well as instructive.

I have had the privilege of working with an excellent group of fellow students. Alex Veidenbaum has taught me much of what I know about compilers for parallel machines and the intricacies of Parafrase. The inhabitants of 223 , and later $302 \mathrm{c} / \mathrm{d}$, have always provided stimulating and amusing conversation.

Finally, I would like to thank my parents and family for their support and faith during the ups, and downs, of my academic career. Without them, this thesis could not have been written. 


\section{T.ABLE OF CONTENTS}

\section{CHAPTER}

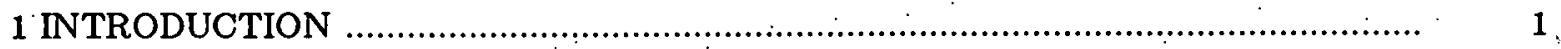

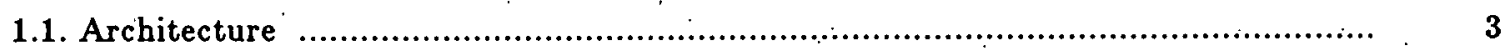

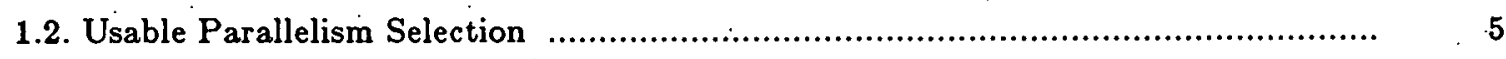

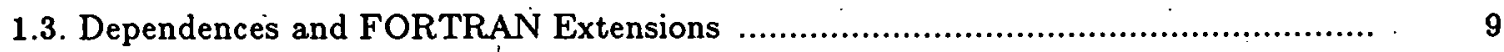

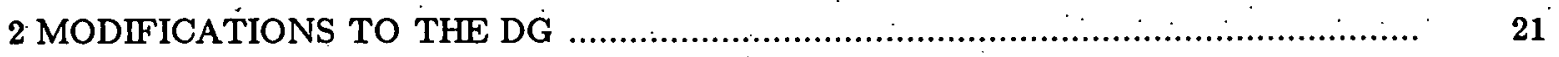

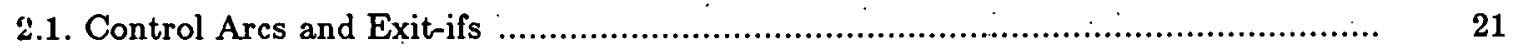

2.2. Nested Loops ................................................................................... $\quad 23$

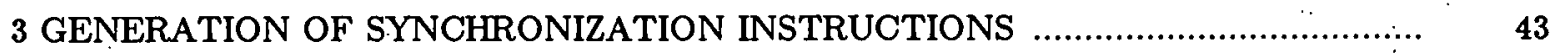

3.1. Synchronization Instructions ........................................................ 43

3.2. Synchronizing Within Blocks of Assignment Statements (BAS) ..................... 45

3.3. Advantages and Disadvantages of the Instruction Sets ............................... 49

3.4. Loop Modifications for Exit-ifs ....................................................... 52

3.5. Adding .Instructions in Loops with Complex Flow of Control ........................... . 54

4 DEPENDENCE ARC ELIMINATION AND DEPENDENCE FOLDING .................

4.1. Introduction ............................................................................ 59

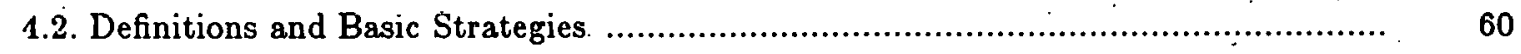

4.3. Building a CPG in a BAS .................................................................. 62

4.4. Dependence Arc Elimination in a BAS .......................................................67

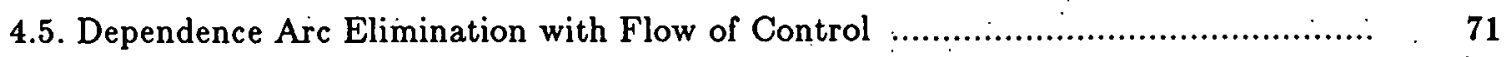

4.6. Optimal Dependence Arc Elimination ................................................. 77

4.7. Dependence Arc Elimination in Nested Parallel Loops ................................. 80

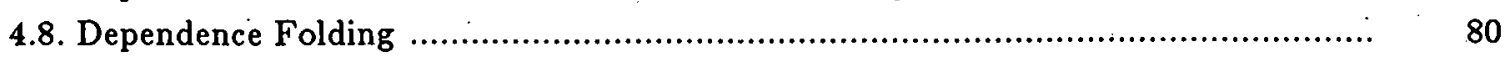

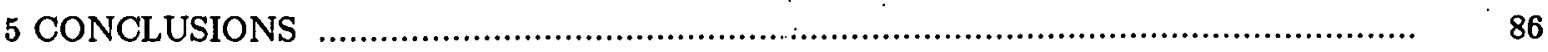

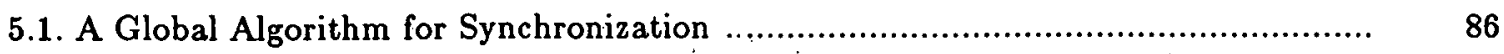

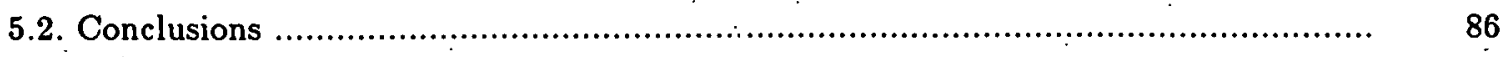

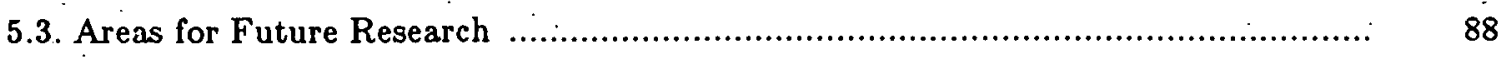

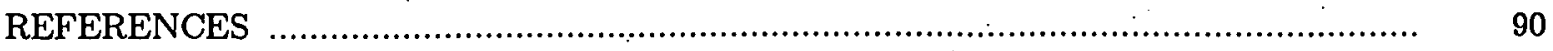




\section{CHAPTER 1}

\section{INTRODUCTION}

The need for faster computing has brought about projects to develop computers with up to several thousand processing units capable of being brought to bear on a single program. These computers seek to exploit two different types of parallelism: extra-loop and intra-loop. Extraloop parallelism assigns program segments containing one or more loops to a processor and runs different segments in parallel. The University of Illinois Cedar project [GKLS83] and the Cray XMP series [Chen83] are the most notable examples of multi-processors exploiting this type of parallelism. Intra-loop parallelism assigns program segments containing one or more iterations, or part of an iteration, to a processor, and thereby runs loop iterations in parallel. Intra-loop parallelism is exploited by the Cedar project computer, the Alliant FX series [Alli85] computers, the NYU Ultra Computer [GGKM83], the Cray XMP using micro-tasking and the Denelcorp HEP-1 [Smit81] among others.

This thesis concentrates on automatic generation of synchronization for intra-loop parallelism. When the body of a loop is executed on several processors, it is often necessary to restrict the order of execution of some statements within the body of the loop so that the results produced by the parallel version of the program are identical to those produced by the serial version of the same program. It is the job of synchronization to enforce these restrictions on the execution order of statements. This thesis develops algorithms for the automatic generation of synchronization instructions. We concern ourselves with FORTRAN DO loop nests that have at most one loop whose parallelism is realized by spreading the loop across multiple processors.

We also assume that parallel loops have already been marked before the invocation of the algorithms in this thesis. The Parafrase compiler [KKLW81], [KLPL81], [Wolf82] that automati- 
cally detects parallel loops, and performs code transformations to enhance the detection of parallel loops, is one example of software that is capable of doing this. Many of the algorithms in this thesis have been implemented in a pass of the Parafrase compiler, but their usefulness is in no way restricted to this compiler.

This thesis contains 5 chapters. The first introduces the architecture of the computer for which architecturally dependent algorithms are targeted. An algorithm is given for determining which loops should be executed as parallel loops on this arrchtecture, to give an optimal running time, when multiple parallel loops exist in a loop nest. It also describes the notation used in this thesis, and provides some definitions of basic terms. Finally, the fundamental data structure used in this thesis, the dependence graph is explained in detail.

In the second chapter we modify the dependence graph to take into account the presence of certain types of control branches present in loops. 'These include branches outside the scope of the parallel loop being synchronized, and serial loops nested within the parallel loop being synchronized. These transformations are largely independent of the target architecture.

The third chapter defines two synchronization instruction sets and shows how synchronization is generated using these. Both the restricted case of adding synchronization instructions when no control branches are present in the parallel loop, and the general case of adding synchronization instructions to loops with arbitrary acyclic flow of control graph are covered.

Thie algorithms of the first three chapters are sufficient to determine where synchronization instructions are needed in a loop, and to place those synchronization instructions in the program. The fourth chapter presents algorithms for minimizing the number of dependences that must be synchronized, a process we refer to as dependence arc elimination: Dependence arc elimination is shown to be NP-hard, and a pseudo-polynomial time algorithim [GaJo79] is given. The transformations of this chapter have architecturally dependent and independent components. 
The fifth chapter gives our conclusions, and areas for further study: It also presents a global algorithm that ties together the use of the other algorithms presented in the earlier chapters.

\subsection{Architecture}

A block diagram of the target machine is shown in Figure 1. Memory access is assumed to be deterministic: eilher memory accesses from processor units take a bounded amount of time, or a processor can suspend itself until all pending stores and fetches are complete.

Synchronization registers are contained within the global memory modules. By reading or writing these registers through the interconnection network, processors can signal and detect the completion of events.

The architecture is capable of supporting two levels of parallelism simultaneously. The first level, achieved by spreading iterations across processors, is referred to as spread parallelism in this thesis, and is designated in programs by the dospread loop construct. It will be con-

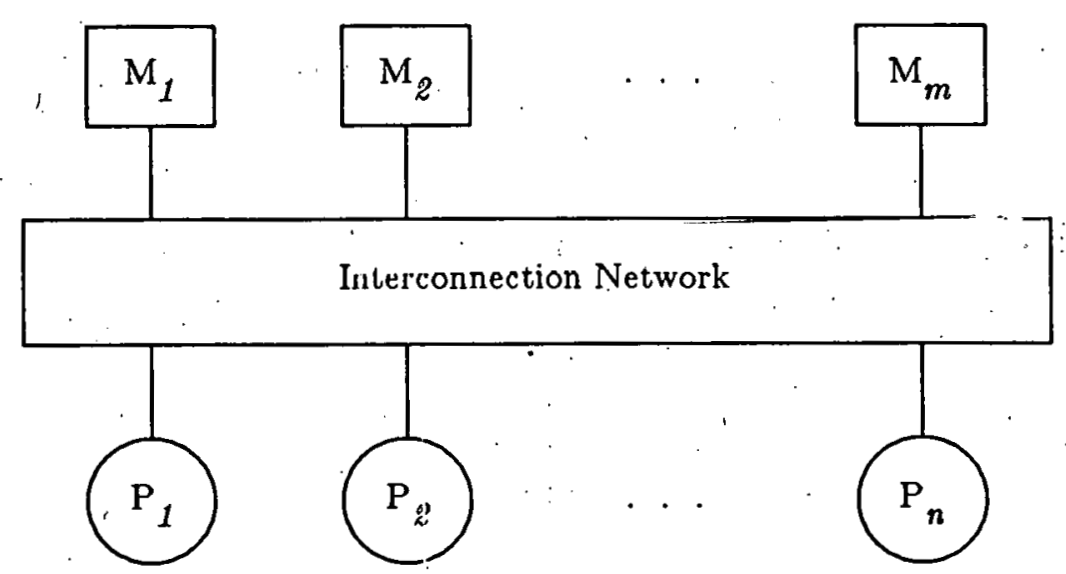

$\mathrm{M}_{i}$ is Global Memory Module $i . \quad \mathrm{P}_{j}$ is Processor $j$.

Figure 1. Systern Architecture 
sidered illegal for a dospread loop to contain a nested dospread loop. The second level is achieved by executing certain statements in vector mode. Statements to be so executed are designated in programs by being enclosed in a dovector loop. Each processor is assumed to have a functional unit capable of executing such statements in vector mode. Section 1.3.2, which discusses FORTRAN extensions, defines these loops in terms of other types of parallel loops found in the literature. An important point to note about the two types of parallelism is that spread parallelism may require synchronization statements be added to the program, whereas vector parallelism will never cause synchronization statements to be introduced. Within a processor, and therefore an iteration', all fetches and stores execute serially.

Dospread loops can be scheduled in different ways. Figure 2a shows how sixteen iterations of a loop would be horizontally 'spread [Jack85] over four processors, and Figure 2b shows how these same iterations would be vertically spread over four processors. This can be accomplished by self scheduling, or by loop blocking. With self scheduling, a processor will query a registei or memory location for the value of the next iteration to be rün. This it will du by lucking, reading, incrementing and unlocking the register or memory location. The value thus read will be the number of the next iteration to be run. This process suffers from potentially serializing the execution of the program.

The other method involves loop blocking. In loop blocking, the dospread loop is made serial and a new dospread loop is placed around the just serialized loop, $L_{0}$. This dospread loop runs from one to the number of processors available, $P$, to execute the loop. For horizontal spreading, the loop bounds of $L_{0}$ are adjusted to: '

$$
\text { do } i:=p \text { to } N \text { by } P
$$

where $p, 1 \leq p \leq P$, is the number of the processor executing $L_{0}$. If vertical spreading was used, then a processor would execute iterations $i, i+1, i+2, \cdots, i+k$. It is of ten the case that the processor executing iteration $i+k+1$ would have to wait until-some statements of all 


\begin{tabular}{|c|c|c|c|c|}
\hline processor $=$ & 1 & 2 & 3 & 4 \\
\hline \multirow[t]{4}{*}{$\mathrm{i}=$} & 1 & 2 & 3 & 4 \\
\hline & 5 & 6 & 7 & 8 \\
\hline & 9 & 10 & 11 & 12 \\
\hline & 13 & 14 & 15 & 16 \\
\hline
\end{tabular}

(a) Horizontal Spreading

\begin{tabular}{c|cccc} 
processor $=$ & 1 & 2 & 3 & 4 \\
$\mathrm{i}=$ & 1 & 5 & 9 & 13 \\
2 & 6 & 10 & 14 \\
3 & 7 & 11 & 15 \\
4 & 8 & 12 & 16
\end{tabular}

(b) Vertical Spreading

Figure 2. Horizontal and Vertical Spreading of Iterations

iterations less than iteration $i+k+1$ finished before beginning execution. Therefore iterations of loops with cross iteration dependences should not be vertically scheduled since this would serialize the loop.

\subsection{Usable Parallelism Selection}

Often, more parallelism will be found in a loop nest than can be utilized by the architecture of this thesis. For example, if there are three nested parallel loops in a program, it will never be possible to execute more than two of them as parallel loops. The inner loop may be executed as a dovector loop, and one outer loop as a dospread loop. The general problem of optimally assigning processors to various parallel loops in a loop nest is not solvable if certain assumptions cannot be made at compile time about the running times of loops. [Poly86] shows that with sume slight resirictions this can be done in polynomial time. The task we give ourselves is much simpler. Because nested dospread loops are'not allowed, we need only examine the parallel running time of each loop in the nest. If the loop nest executes faster by having this loop run in parallel and all surrounding and enclosed loops run scrially, then the optimal 
schedule for the loop nest will mark this loop as parallel and the others as serial. Since only one loop is running in parallel at a time, all available processors can be given to this loop. We make no assumptions about the number of processors available to run a loop, nor do we make any attempt at determining the optimal number of processors to execute a loop nest. We also do not attempt to do loop interchanging [Wolf82]. We simply decide, given a loop nest and some number of processors, which loops in the nest to run in parallel.

As will be seen, to do this requires visiting each loop only once. Therefore, scheduling for our architecture can be done in $O(n)$ time, where $n$ is the number of loops in the loop nest.

The loop nest, which serves as input to this algorithm, is assumed to already have all parallel loops marked as such. Loops that can be executed in vector mode are marked as dovector, and all other parallel loops are marked as dospread.

A loop nest, such as is found in Figure 3a, can be represented as a tree, such as the one found in Figure 3b. Attached to each node are three times. The first, $S S$, gives the time it takes for the loop to execute serially, and for all loops nested within it to execute serially. The sccond, $S P$, gives the time for the loop to execute serlally, and for all loops nested within il to execute in the fastest possible manner. That is, the time for the optimal schedule of the subnest is used. The third, $P S$, gives the time for the node to execute in parallel, and all loops nested within it to exccutc serially. Each node is also marked spread or serial, depending on whether the optimal schedule at this point requires the loop to be run as a dospread loop or not.

$\bar{F}$ igure 3 b shows the times computed for the nodes $b, c, d, e$ and $f$. These have already been computed by applying the bottom up algorithm of Figure 4 . We compute the times for a loop by adding the appropriate times for nested loops to the time for in -line code in the loop. (In-line code is code that is nested within the loup being timed, but is not contained in any other more deeply nested loops.) This is then multiplied by the number of iterations in the loop, and for dospread loops, divided by the number of processors assigned to execute the loop. If the 


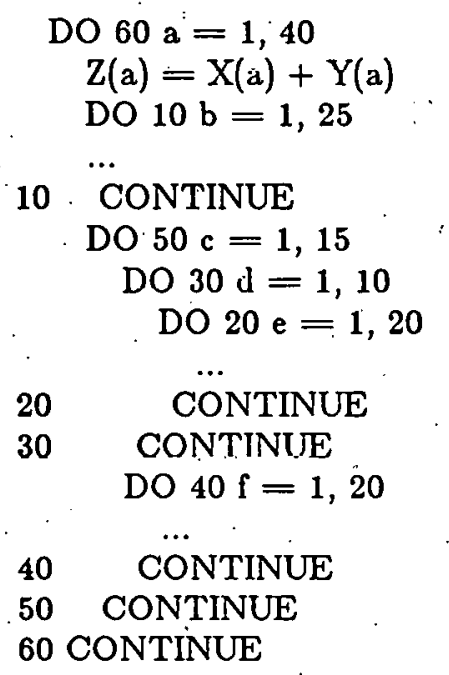

(a) A Loop Nest to be Scheduled

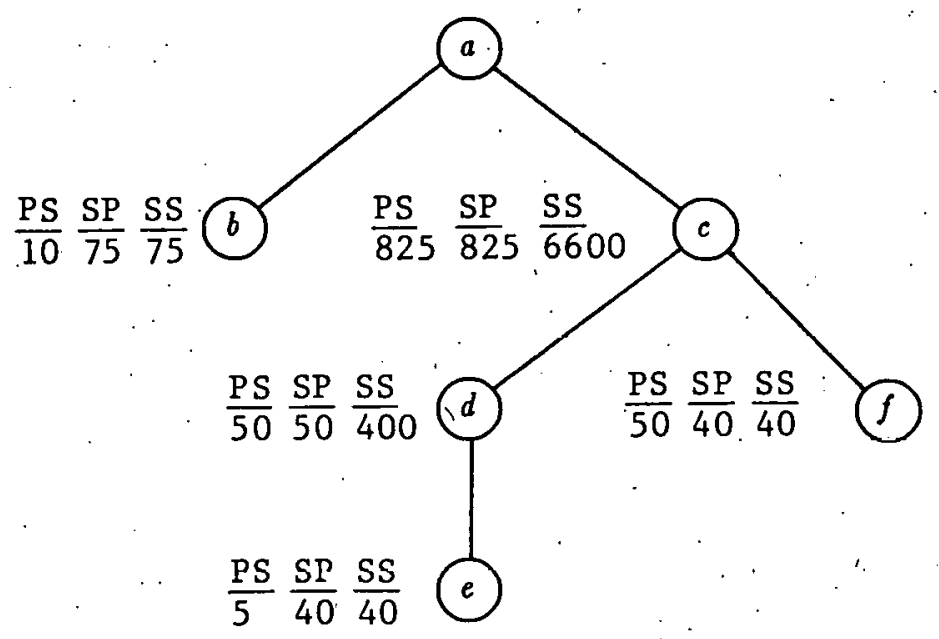

Figure 3. Loop Scheduling Example

dospread has lexically backward dependences, or has an exit-if, then the execution of iterations cannot totally overlap, and running time of the loop must be increased. It will be assumed that a value, delay [Cytr84] can be found that can be used to determine the true running time of the loop. For our example we will assume that no lexically backward dependences exist, i.e. that 
delay $=0$. Other functions, $[$ Poly86] and [Cytr84], can be used to calculate the running time of a loop.

We now compute the time at node $a$. The $S S$ time is the sum of the $S S$ times for all. immediately nested loops; i.e. $b$ and $c$, plus the time for the in-line code. We will assume in-line code takes one time unit per statement to execute, times the number of iterations in the loop.

$F_{p}-$ a timing function such as found in [Poly86] and [Cytr84]

\#processors - the number of processors assigned to execute the loop nest

All nodes are initially marked as do or dovector

Visit each node $n$ using a post-order traversal

$S S[n]=$ execution time for the serial execution of all code, including nested loops

$S P[n]=$. execution time for in line code to execute serially, and nested loops in parallel if possible

$P S[n]=$ execution time for inline code to execute in parallel, and nested loops to execute serially

foreach child $c$ of $n$ do

$S S[n]:=S S[n]+S S[c]$

if $c$ is marked as spread then

$S P[n] .=S P[n]+P S[c]$

else

$$
S P[n\rfloor-S P\lfloor n\rfloor+S P\lfloor c]
$$

$P S[n]=F_{\dot{p}}(S S[n]$, \#processors, delay $)$

if $P S[n] \leq S P[n]$ then

mark loop as spread

Visit each node $n$ using a preorder traversal of the tree

if an ancestor of $n$ is marked spread then

mark $n$ as serial.

if $n$ is a leaf then

if $n$ was a dovector loop then

if $n$ is marked opread then

run $n$ as a dospread loop with enclosing

statments executed as vector operations.

else

run $n$ as a dovector loop

Figure 4. Algorithm for Scheduling Loops 
Thus the $S S$ time is $(6,600+.75+1) \cdot 40$, or 267,040 . The $S P$ time is computed as above, except now. we use the $P S$ times for each of the immediately nested loops that is marked as dospread, and the $S P$ time for each of the loops that is not marked as dospread. This leads to an $S P$ time of 33,440 . And the $P S$ time is computed like the $S S$. time, except that we divide the final result by the number of processors assigned to the loop. The PS time is 33,380 .

Since the time for running $a$ in parallel and its children serially is less than or equal to the time for running $a$ serially and its children in parallel, we should run $a$ as a dospread loop, and its children serially. This would be shown by marking $a$ as dospread, and its children as serial.

\subsection{Dependences and FORTRAN Extensions}

This section seeks to both define those terms that are peculiar to this thesis, and to define and give some background on those terms that are more widely used. The discussion of the more general terms will of necessity be more abbreviated than that contained within the mentioned references.

\subsubsection{Dependences and Dependence Gräphs}

This section defines what various types of dependences are, and shows how the dependence structure of a program can be represented by a directed graph called a dependence graph. We first define dependences and their properties, and give examples. We then show how to construct a dependence graph.

\subsubsection{Definitions}

We often refer to statements within the loop being synchronized. $S_{i}$ refers to statement $i$ in a FORTRAN source program. If $i<j$, then $S_{i}$ lexically precedes $S_{j}$. $L_{i}$ refers to some loop nested within the dospread loop being synchronized. Sometimes a nested loop, $L_{i}$, will be broken into several loops. These will be called $L_{i}{ }^{1}, L_{i}{ }^{2} \cdots L_{i}^{n}$, where $L_{i}^{k}$ is the lexically $k$ 'th loop. 
Dependences that must be synchronized fall into four categories: flow, anti, output [Kuck78] [Wolf82] and control [Kuck78]. Figure 5a, Figure 6a, Figure 7a and.Figure 8a, respectively, give examples of these. Flow, anti and output dependences are collectively know as data dependences because they express a constraint on the flow of data through the program. Each of the data dependences is defined using the $I N$ and $O U T$ sets of the statements involved in the dependence.

$\operatorname{OUT}\left(S_{i}\right)$ is the set of the output variables of $S_{i}$. In an assignment statement this would be the left hand side, or $L H S$, variable [AhU17.7]. $I N\left(S_{i}\right)$ is the set of the input variables of $S_{i}$. In an assignment statement these would be the right hand side, or RHS, variables [AhUl77].

A flow data dependence from $S_{i}$ to $S_{j}, S_{j} \delta S_{i}$, exists if $\alpha \in O U T\left(S_{i}\right), \alpha \in I N\left(S_{j}\right)$, and the value of $\alpha$ used in $S_{j}$ could come from $S_{i} . S_{i}$ is the source of the dependence, and $S_{j}$ is the sink of the dependence.

DO $10 \mathrm{I}=1, \mathrm{~N}$
$\mathrm{~A}(\mathrm{I})=\mathrm{B}(\mathrm{I})+\mathrm{C}(\mathrm{I})$
$\mathrm{D}(\mathrm{I})=\mathrm{A}(\mathrm{I}-1)+\mathrm{B}(\mathrm{I}) \quad \cdot \quad S_{1}$
10 CONTINUE

(a) Fluw Dala Dependence

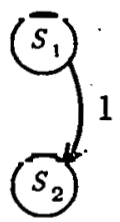

(b) DG for (a)

Figure 5. F'low Data Dependence Example 


$$
\begin{gathered}
\text { DO } 10 \mathrm{I}=1, \mathrm{~N} \\
\mathrm{~A}(\mathrm{I})=\mathrm{B}(\mathrm{I})+\mathrm{C}(\mathrm{I}) \\
\mathrm{B}(\mathrm{I}-1)=\mathrm{C}(\mathrm{I})+\mathrm{D}(\mathrm{I}) \\
10 \text { CONTINUE }
\end{gathered} \quad \begin{aligned}
& S_{1} \\
& S_{2}
\end{aligned}
$$

(a) Anti Data Dependence

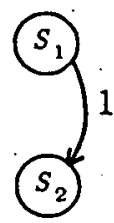

(b) DG for (a)

Figure 6. Anti Data Dependence Example

$$
\begin{aligned}
& \text { DO } 10 \mathrm{I}=1, \mathrm{~N} \\
& \mathrm{~A}(\mathrm{I})=\mathrm{B}(\mathrm{I})+\mathrm{C}(\mathrm{I}) \\
& \text { A }(\mathrm{I}-1)=\mathrm{B}(\mathrm{I})+\mathrm{D}(\mathrm{I})
\end{aligned}
$$

(a) Output Data Dependence

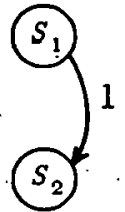

(b) DG for (a)

Figure 7. Output Data Dependence Example 


$$
\begin{gathered}
\text { DO } 10 \mathrm{I}=1, \mathrm{~N} \\
\text { IF (A(I).GT.B(I)) GOTO } 10 \quad: S_{1} \\
\text { X(I)=C(I)+D(I). } \\
\text { 10 CONTINUE }
\end{gathered}
$$

(a) Control Dependence

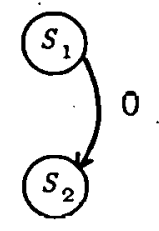

(b) DG for (a)

Figure 8. Control Dependence Example

An anti-data dependence from $S_{i}$ to $S_{j}, S_{j} \bar{\delta} S_{i}$, exists if $\alpha \in \operatorname{IN}\left(S_{i}\right), \alpha \in \operatorname{OUT}\left(S_{j}\right)$, and the value of $\alpha$ is used in $S_{i}$ before a new value of $\alpha$ is stored in $S_{j} . S_{i}$ is the source the dependence, and $S_{j}$ is the sink of the dependence. $S_{i}$ must be executed before $S_{j}$.

A output data dependence from $S_{i}$ to $S_{j}, S_{j} \delta^{\circ} S_{i}$, exists if $\alpha \in \operatorname{OUT}\left(S_{i}\right), \alpha \in \operatorname{OUT}\left(S_{j}\right)$, and the value of $\alpha$ in $S_{j}$ could be stored after the value of $\alpha$ is stored in $S_{i}$. S $S_{i}$ is the surute of the dependence, and $S_{j}$ is the sink of the dependence.

A control dependence from $S_{i}$ to $S_{j}, S_{j} \delta^{c} S_{i}$, exists if whether $S_{j}$ executes depends the execution of $S_{i} . S_{i}$ is the source of the dependence, and $S_{j}$ is the sink of the dependence.

That some statement is the source of a dependence will often be denoted by substituting so for the subscript $i$ in a statement specification. A sink is denoted by substituting si. Therefore, $S_{80}$ refers to the statement that is the source of some dependence. If $s i>s 0^{\circ}$, i.e. the sink of the dependence is lexically forward of the source, the dependence is a lexically forward dependenec, or $L F D$. If $s i \leq s o$, i.e. the sink of the dependence is lexically prior to the dependence, the dependence is a lexically backward dependence, or $L B D$. 
The dependence distance, $\Delta$, is the number of iterations spanned by the dependence. In Figure 9, we see the iteration space [Wolf82] of Figure 5a, with the dependences drawn in. Here the dependence distance is one. In general, the dependence distance from one occurrence of the array variable $A, A\left(f_{1}(I)\right)$, to another occurrence, $A\left(f_{2}(I)\right)$, is $\Delta=f_{1}(I)-f_{2}(I)$; where $I$ is the index variable of the loop. Depending on the functions, $f_{1}$ and $f_{2}$, the distance may either be constant or changing. If $\Delta$ is the distance of some dependence, then $\Delta_{\min }^{L}$ is the minimum value of all $\Delta \mathrm{s}$ in a loop $L$, and $\Delta_{m a x}^{L}$ is the maximum value of all $\Delta \mathrm{s}$ in loop $L$.

Often it is not necessary to know the distance of a dependence: its direction alone suffices. The direction can be determined from the relative values of the respective subscript fuactions $f_{\theta 0}$ and $f_{\theta i}$, for the source and sink of the dependence. If $I$ is an index value for'some loop, then the direction, $\gamma$, of the dependence on that loop is $f_{a i}(I) \gamma f_{\circ o}(I)$, where $\gamma \in\{<,=,>,<=$, $>=,\langle\rangle,\langle\doteq>$ \}. In other words, if $\gamma$ is ' $<$ ", the dependence is from an earlier iteration to a later iteration. If $\gamma$ is ' $>$; the dependence is from a later iteration to an earlier one. And if $\gamma$ is $'='$, the dependence is within the same iteration.

$$
\begin{aligned}
\mathrm{i} & =1 \\
& =2 \\
& =3 \\
& =4 \\
& =5 \\
& =6
\end{aligned}
$$

Figure 9. Iteration Space of Figure 1a 


\subsubsection{The Importance of Dependences}

As stated before, a dependence is a relation between the source and sink of a dependence. This relation says that the source of the dependence must execute before the sink of the dependence if the parallel execution of the program is to be the same as the serial execution of the program. Therefore, the sink of a dependence must wait until the source executes before executing. It is the purpose of synchronization to force this. Thus, regardless of the synchronization scheme used, some action must be taken before or during the sink of a dependence to insure that it does not execute until the source has. Likewise, the execution of the source of a dependence must allow the sink to eventually execute.

\subsubsection{Building the Dependence Graph}

A dependence $\delta$ is a relation between two statements, $S_{80}$ and $S_{8 i}$. This relation can be expressed as an arc on a graph whose nodes are statements. The dependence $\delta=\left(S_{\bullet 0}, S_{e i}\right)$ can be represented by a directed arc whose head is at $S_{a n}$ and whose tail is at $S_{a i}$. Such a graph will be referred to as a dependence graph, or $D G$. The next section explains how to build a DG. Figure $5 \mathrm{~b}$, Figure $6 \mathrm{~b}$, Figure $7 \mathrm{~b}$ and Figure $8 \mathrm{~b}$ give examples of DGs.

As has been mentioned, the primary tool used to generate synchronization for programs is the DG. The DG used for synchronizing programs with the architecture assumed in this thesis need only be a statement level DG. Nested loops are treated as complex statements, and the entire nested loop is represented as a single node on the DG. As well, the DG need only include dependences that have a dependence direction component of ' $<$ ' for the loop being synchronized. The direction components of other loops may be ignored, since those loops are serial and therefore cannot cause synchronization instructions to be generated. The reasons for this, which are discussed shortly, are entirely dependent on the architecture we have assumed. Other architectures may require the inclusion of dependences with ' $=$ ' and ' $>$ ' direction components, or require an atom level DG. We note that if the dependence we leave out were included in the DG 
they would be removed by the dependence arc elimination algorithms of Chapter 4 .

Control dependences, with the exception of exit-ifs (a control branch whose target is outside the dospread loop), have an ' $=$ ' dependence direction. Therefore control dependences are not added to the DG at this time. In Chapter 2, we augment the DG with control dependences for exit-ifs. Thus when the DG is built, only flow, anti and output dependences are included.

\subsection{Justification for a Statement Level DG}

The use of a statement level DG for determining the synchronization points implies that synchronizing a dependence from an atom in some statement to another atom in some statement synchronizes all dependences from the former statement to the latter statement, assuming the dependence distances cre the same.

For example, consider the program in Figure 10. There exists a flow dependence from $A(I)$ in $S_{1}$ to $A(I-1)$ in $\dot{S}_{2}$, and an anti-dependence from $B(I)$ in $S_{1}$ to $B(I-1)$ in $S_{2}$. Since statement $S_{2}$ cannot begin executing until all fetches and stores in statement $S_{1}$ have finished,

$\begin{array}{ccc}\text { DOSPREAD } 10 \mathrm{I}=1, \mathrm{~N} \\ \mathrm{~A}(\mathrm{I})=\mathrm{B}(\mathrm{I})+\mathrm{C}(\mathrm{I}) & S_{1} \\ \mathrm{~B}(\mathrm{I}-1)=\mathrm{A}(\mathrm{I}-1)+\mathrm{C}(\mathrm{I}) & S_{2} \\ 10 \quad \text { CONTINUE } & \end{array}$

(a) Loop with Multiple Dependences with equal $\Delta$ s between Two Statements

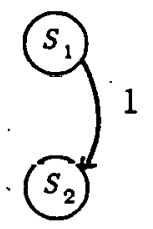

(b) DG for the Program in (a)

Figure 10. Multiple Dependences and Equal $\Delta \mathrm{s}$ 
$S_{2}$, cannot distinguish between the store of $A(I)$ and the fetch of $B(I)$. Thus the statement can be viewed as a single indivisible entity, and represented by a single node on the DG. Figure 10b shows the DG of the loop of Figure 10a.

Complications arise, however, when the dependences have different distances, as in the program of Figure 11a. We again have an anti-dependence of distance one from $B(I)$ in $S_{1}$ to $B(I-1)$ in $S_{2}$. The flow dependence from $A(I)$ in $S_{1}$ to $A(I-2)$ in $S_{2}$ has a distance of two. If the DG is to be a statement level DG can both dependences from $S_{1}$ to $S_{2}$ be merged? In general, the answer is no. [LiZh85] proves this for one synchronization instruction set. Figure 11b shows the DG for the the program of Figure 11a.

This is particularly bad when the dependence distance between two statements is not a constant function of the loop index. If the dependence distance is not a constant function of the loop index, then arcs must be generated for every possible value that the function can take. If the upper bound of the loop is not known, then it might not be possible to generate all these

10

DO $10 \mathrm{I}=1, \mathrm{~N}$

$$
\begin{array}{ll}
\mathrm{A}(\mathrm{I})=\mathrm{B}(\mathrm{I})+\mathrm{C}(\mathrm{I}) & S_{1} \\
\mathrm{~B}(\mathrm{I}-2)=\mathrm{A}(\mathrm{I}-1)+\mathrm{C}(\mathrm{I}) & S_{2}
\end{array}
$$
CONTINUE

(a) Program with Multiple Dependences with non-equal $\Delta$ s Between Two Statements

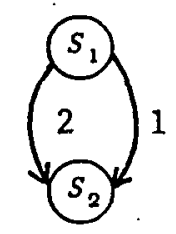

(b) DG for the Program in (a)

Figure 11. Multiple Dependences and Non-equal $\Delta \mathrm{s}$ 
arcs, making synchronization for certain instruction sets impossible. As shall be seen in Chapter 3, when the testset instruction set is used, the distance can be assumed to be $\Delta_{\min }^{L}$, the shortest dependence from one statement to another. Because we are treating the more general case at this point, we shall assume that all dependence arcs are placed in the DG. Furthermore, if synchronization cannot be generated for a loop, we assume that the loop was marked as serial either by other passes or by a prescan pass with a knowledge of synchronization.

\subsection{Justification for Including Only ' $<$ ' and ' $<=$ ' Dependences'}

If a dependence does not have a ' $<$ ' direction component it must have either ' $>$ ' or ' $=$ ' direction components: If the dependence direction is ' $=$ ' the dependence is completely within an iteration. Since we assume that a single iteration always runs on a single processor, and fetches and stores within a processor execute serially the dependence is forced to be honored by the hardware. If the hardware did not constrain the order of fetches and stores within a single processor, then '=' dependences would have to be included in the DG.

If a dependence has a ' $>$ ' direction then the source of the dependence is in a later iteration of the dospread loop than the sink. The only way such a dependence can be violated is for multiple invocations of the dospread loop to be active simultaneously. This, however, requires that a loop surrounding the dospread loop be parallel and executing on multiple processors. The architecture, however, only allows one level of spread parallelism, so this cannot occur. If multiple levels of spread parallelism were allowed, ' $>$ ' dependences would alșo have to be included in the DG. The synchronization instruclion semantics would also have to be altered to allow a synchronization etatement to be associated with a particular dospread loop within a loop nest, and the sign of $\Delta$ in the test instruction would have to be taken into account.

If we need to construct a DG for a loop nested within a dospread loop to perform the transformations of Section 2.3.2 then we should include all dependences regardless of direction. 


\subsubsection{FORTRAN Extensions}

Programs that are the object of the' automatic synchronization techniques are written in extended FORTRAN 66. Four new statements are needed to specify synchronization actions and objects. These statements are not present in the input language, but are found only in the output language from synchronization. The new statements are test, set, testset and quit. They are explained in greater detail in Chapter 2.

The input' language includes twō new loop lypes, luspread and dovector.

A dospread loop is one that is spread across several processors to take advantage of intraloop parallelism. A dospread́ loop can be a doall [Davi81], [LuBa80], a forall [Padu79], or a doacross [Cytr84] loop. A doall loop is any loop that has no cross-iteration dependences, i.e. any dependences that exist have a direction of ' $=$ '. A forall loop may have cross itcration dependences; but they must all be from a statement to a lexically forward statement, i.e. LFDs. A doacross loop is a loop whose iterations may not all start at the same time. Instcad, each iteration must wait some amount of time after the last iteration has started before itself starting. This wait will be caused, in thls thesls, by symcluvinization. The wait is nosessitateft pilitier hy a branch out of the body of the loop, or by a LBD. Figure 12a gives an examples of a doall looper, and Figure 12b gives the DG for the doall loop. Figure 13a, Figure 13b, Figure 14a and Figure $14 \mathrm{~h}$ give the same for forall and doacross loops respectively.

The doveclor loop is one that can potentially be run as a set of veclur instructions. Any loop that has been determined to be a doall or a fur ull loop is a candidato to bo run as a dnmer.tor loop. 


$$
\begin{gathered}
\text { DO } 10 \mathrm{I}=1, \mathrm{~N} \\
\mathrm{~A}(\mathrm{I})=\mathrm{B}(\mathrm{I})+\mathrm{C}(\mathrm{I}) S_{1} \\
\mathrm{D}(\mathrm{I})=\mathrm{A}(\mathrm{I})+\mathrm{B}(\mathrm{I}) S_{2} \\
10 \text { CONTINUE }
\end{gathered}
$$

(a) Doall Loop

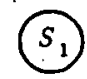

$S_{2}$

(b) DG for (a)

Figure 12. Doall Loop Example

$\begin{array}{cc}\mathrm{DO} 10 \mathrm{I}=1, \mathrm{~N} & \\ \mathrm{~A}(\mathrm{I})=\mathrm{B}(\mathrm{I})+\mathrm{C}(\mathrm{I}) & S_{1} \\ \mathrm{D}(\mathrm{I})=\mathrm{A}(\mathrm{I}-1)+\mathrm{C}(\mathrm{I}) & S_{2} \\ 10 \mathrm{CONTINUE}\end{array}$

(a) Forall Loop

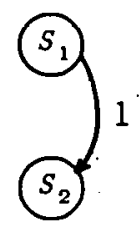

(b) DG for (a)

Figure 13. Forall Loop Example 


$$
\begin{aligned}
& \text { DO } 10 . \mathrm{I}=1, \mathrm{~N} \\
& \mathrm{~A}(\mathrm{I})=\mathrm{B}(\mathrm{I})+\mathrm{C}(\mathrm{I}-1) \\
& \mathrm{C}(\mathrm{I})=\mathrm{A}(\mathrm{I}-1)+\mathrm{C}(\mathrm{I}) S_{1} \\
& \mathrm{E}(\mathrm{I})=\mathrm{D}(\mathrm{I})+\mathrm{C}(\mathrm{I}-2) \\
& 10 \mathrm{CONTINUE}
\end{aligned}
$$

(a) Doacross loop

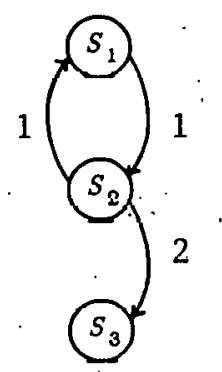

(b) DG for (a)

Figure 14. Doacross Loop Example 


\section{CHAPTER 2}

\section{MODIFICATIONS TO THE DG}

In the previous chapter the loops to be synchronized were marked as dospread, and a DG was constructed for those loops. In this chapter this DG is modified to take into account certain control structures in the DG, namely branches whose targets are outside of the loop and nested loops. The DG so modified is then ready to be used to place synchronization instructions in the source loop using the algorithms of the Chapter 3.

\subsection{Control Arcs and Exit-ifs}

The targets of GOTO statements within a dospread loop can either be to other statements within the loop or to statements outside the loop. If the former, then the GOTO will either be lexically forward or lexically backward. If it is lexically forward nothing needs to be done to the DG; the actions described in Chapter 3 will insure that the execution of the loop proceeds safely. If the GOTO is lexically backward, we will assume that all enclosing loops are serial. This is a consequence of dependence testing being extremely difficult when backward GOTOs are present in a loop. If the conditions governing the backward GOTO are simple enough the backward G.OTO can be converted into a DO loop.

GOTOs that are to targets outside the loop, and their associated IF statement, are referred to as exit-if s. When exit-ifs are present the DG must be modified to reflect this.

Figure 15a shows a dospread loop that contains an exit-if statement. The exit-if causes two problems. The first problem is that the first statement of some iteration $i+1$ of the dospread loop should not begin executing until the exit-if of iteration $i$ has executed and taken the false path. The order of execution that must be maintained is shown in Figure 15b. 


\begin{tabular}{|c|c|}
\hline . & $\begin{array}{l}\text { DOSPREAD } 10 \mathrm{I}=1,4 \\
\mathrm{~A}(\mathrm{I})=\mathrm{B}(\mathrm{I})+\mathrm{C}(\mathrm{I}) \\
\mathrm{IF}(\mathrm{A}(\mathrm{I}-1) \cdot \mathrm{GT} \cdot 4.0) \text { GOTO } 20 \\
\mathrm{D}(\mathrm{I})=\mathrm{A}(\mathrm{I}-1)+\mathrm{F}(\mathrm{I})\end{array}$ \\
\hline 10 & CONTINUE \\
\hline 20 & $X(I)=Y(I)+Z(I)$ \\
\hline
\end{tabular}

(a) Loop Containing an Exit-if

\begin{tabular}{|c|c|c|c|c|}
\hline$i=$ & 1 & 2 & 3 & 4 \\
\hline$t=1$ & $S_{1}$ & & & \\
\hline-2 & $S_{\text {; }}$ & & & \\
\hline$=3$ & $S_{3}$ & $S_{1}$ & & \\
\hline & & $\dot{S}_{2}^{2}$ & $S_{\mathrm{i}}$ & \\
\hline$=6$ & & & $S_{2}$ & \\
\hline $\begin{array}{l}=7 \\
=8\end{array}$ & & & $S_{3}$ & $\begin{array}{l}S \\
S\end{array}$ \\
\hline$=9$ & & & & $S_{3}$ \\
\hline
\end{tabular}

(b) Execution Order of the Loop of (a)

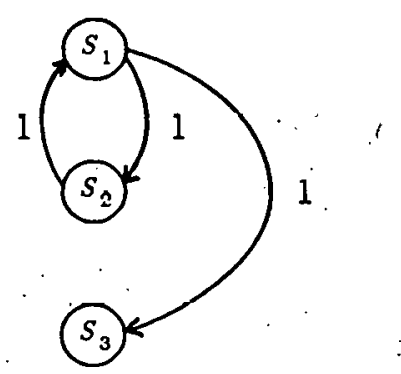

(c) Modified DG of the Loop of (a)

Figure 15. Exit-If Example: DG Modification

The second problem arises when the true path of an exit-if in iteration $i$ is taken, and the loop should terminate. When this happens the processor running the exiting iteration should wait until all iterations less than $i$ have completed, and then terminate the loop. 
The first problem can be solved by modifying the DG and is dealt with here. The second problem requires a change in the source code of the program and some knowledge about the synchronization instruction set being used, and is dealt with in Chapter 3.

We now consider the first problem. A LBD with distance 1 is inserted into the DG from the exit-if statement to the first statement of the loop body that changes the value of any variable used outside that iteration. A control dependence actually exists from the exit-if statement to every statement in the loop. With the architecture of this thesis, all dependence arcs but the one we add will be eliminated by the algorithms of Chapter 4. Therefore we do not need to include these dependence arcs in the DG. Because this dependence will be satisfied by synchronization, iteration $i+1$ cannot execute any statements that will alter any data used outside the loop until iteration $i$ has signaled that it is not going to exit the loop. Since it is the execution of the next iteration that is affected, the distance of the control dependence is one. Figure 15c shows a modified DG where the dashed line is the added LBD.

Figure 16 gives the algorithm for this.

\subsection{Nested Loops}

As we mentioned, only one level of spread parallelism is allowed in the loop nest to be synchronized. This dospread loop can, however, have other loops nested within it. As explained previously, these nested loops are represented as a single node in the DG. If a dependence is lexically forward and its source and sink are not contained within the same nested loop, then the

foreach exit-if branch $S_{E}$ do place a dependence from $S_{E}$ to $S_{1}$ in the $\dot{D} G$

Figure 16. Exit-if DG Modification Algorithm 
DG can be left unchanged. Consider, however, the program of Figure 17a, and its DG in Figure 17b. The dospread loop is effectively serialized by the presence of the dependence cycle on $L_{1}$. The next two sections show how the DG can be modified to recover some or all of the parallelism inherent in a loop nest.

\subsubsection{Synchronization within Nested Vector Loops}

If the complete inner loop nest consists of vector loops then we can fully distribute [Wolf82] the inner loops over their statements: The synchronization instructions can then be placed between the resulting fully distributed loops: Such a loop nest is shown in Figure 17a, and its DG in Figure $17 \mathrm{~b}$.

By fully distributing the inner loops, we get the loop nest of Figure $17 \mathrm{c}$, and.its DG, shown in Figure 17d. This is the approach to take if loops are to operate on vectors, since they will execute as an atomic vector operation. Depending on the overheads associated with vector operations, and the length of vectors, it might be desirable to use loop splitting, discussed in the. next section, along with loop distribution.

\subsubsection{Splitting Non-Distributable Loops}

The second method of dealing with nested loops involves splitting the offending loop into sub-loops, and placing synchronization instructions between the sub-loops. This splitting up of the iteration space of the loop and placing of the synchronization instructions will be referred to as loop splitting. A sub-loop is a loop that executes a set of adjacent iterations of the original loop.. For example, a loop that has iterations from 1 to 1000 might be split into two sub-loops, the first of which executes iterations 1 to 500 , the second iterations 501 to 1000 . Loop splitting differs from loop distribution in that in loop splitting each sub-loop contains all the statements of the original loop, but only part of the iterations. In loop distribution, each distributed loop contains all the iterations of the previous loop, but only part of the statements. 


\section{DOSPREAD $20 \mathrm{I}=1, \mathrm{~L}$ DOVECTOR $10 \mathrm{~J}=1, \mathrm{~N}$$$
\mathrm{A}(\mathrm{I}, \mathrm{J})=\mathrm{B}(\mathrm{I}, \mathrm{J})+\mathrm{C}(\mathrm{I}, \mathrm{J})
$$$$
\text { CONTINUE }
$$$$
\mathrm{D}(\mathrm{I}, \mathrm{J})=\mathrm{A}(\mathrm{I}-1, \mathrm{~J}-1)+\mathrm{B}(\mathrm{I}, \mathrm{J})
$$ \\ 10 CONTINUE \\ $L_{1}$ \\ 20}

(a) Loop Nest With Inner Vector Loops

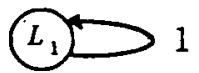

(b) DG for the Loop Nest of (a)

DOSPREAD $30 \mathrm{I}=1, \mathrm{~L}$

DOVECTOR $10 \mathrm{~J}=1, \mathrm{M}$

$A(I, J)=B(I, J)+C(I, J)$

CONTINUE

10

DOVECTOR $20 \mathrm{~J}=1 ; \mathrm{N}$

20

$\mathrm{D}(\mathrm{I}, \mathrm{J})=\mathrm{A}(\mathrm{I}-1, \mathrm{~J}-1)+\mathrm{B}(\mathrm{I}, \mathrm{J})$

$L_{1}^{1}$

$L_{1}^{2}$

30

\section{CONTINUE}

CONTINUE

(c) Loop Nest with Inner Loops Fully Distributed and Synchronization Added

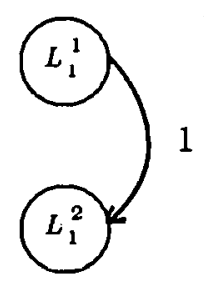

(d) Final DG for (c)

Figure 17. Dependence within a Nested Vector Loops

Loop splitting will allow execution of iterations of the inner loop to be overlapped. Thus the inner loop is executed in parallel. Since the inner loop is executed in parallel, dependences on the inner loop may be violated if not properly synchronized. [Bane79] and [Wolf82] discuss dependence testing and the construction of DGs for multiply nested parallel loops in detail. For 
our purposes, we can assume that a DG for the inner loop can be built in the same manner as for the the outer dospread loop, except that directions of all dependences must be included since there is an outer parallel loop.

When constructing a DG for a dospread loop, only ' $<$ ' direction components are allowed. This means that all dependence distances are positive. When constructing a DG for a nested. loop, it is possible that ' $=$ ' and ' $>$ ' dependence directions exist. This means that dependence distances may be zero and negative. When determining the value of $\Delta_{\text {max }}^{\text {neted }}$ for a nested loop the sign of the distance will be ignored. Therefore, if $\Delta_{1}^{\text {nested }}=-4$, and $\Delta_{2}^{\text {neated }}=3$, then $\Delta_{m x x}^{\text {neated }}=-4$

The goal of loop splitting is to arrange the DG in such a way that synchronizing the dependences will cause both the dependences on the outer dospread and inner serial loop to be honored. Dependences on the outer loop will be honored by the distance attached to the dependences added to the DG during loop-splitting. Dependences on the inner loop will be honored because of the location of the dependences in the DG, which will not allow a sub-loop $L_{k}$, in some iteration $i$ of the outer dospread loop, to begin executing until all sub-loops that generate data for oub loops $L_{k}$ havo oomploted.

Figure 18a shows a loop nest whose nested inner loop is a a candidate for loop splitting. Figure 18b gives the DG for the dospread I loop, and Figure $18 \mathrm{c}$ gives the DG for the inner nested $J$ loop. Figure 18d shows the loop nest of Figure 18a with the inner loop split. Dependence arcs have been drawn that represent represent dependences between the nested loops that must be satistied. Figure 188 shows a $D G$ for the loop of Flgure 18d with the sub-loops represented as a single node for compactness. If $L_{k}$ is a sub-loop, and the sink of such a dependence, then it can be easily determined what sub-loop $L_{k}+$ offoet is the source. Offset is a function of the minimum dependence distance, $\Delta_{\max }^{J}$, on the nested $J$ loop, and the maximum number of iterations, $\iota$, in any sub-loop. The formula is: 


$$
\text { offset }:=\left\lceil\frac{\iota-\Delta_{\max }^{J}}{\iota}\right\rceil-1
$$

A proof is given in Figure 19.

Figure 20 shows values for of $f$ set obtained for different ranges of $\Delta_{\max }^{J}$ with different dependence directions. The values have been derived from the formula for of $f$ set. A more intuitive explanation of these values follows:

An iteration dependence graph (DG) will be used in the examples that follow. An DG is like a DG except that nodes on the graph are iterations, not statements, and arcs represent dependences between iterations and not between statements. An IDG can be created by merging

$\begin{array}{ccc} & \text { DOSPREAD } 20 \mathrm{I}=1, \mathrm{~N} \\ & \text { DO } 10 \mathrm{~J}=1, \mathrm{~N} & L_{1} \\ & \mathrm{~A}(\mathrm{I}, \mathrm{J})=\mathrm{B}(\mathrm{I}, \mathrm{J})+\mathrm{C}(\mathrm{I}, \mathrm{J}) & S_{1} \\ 10 \quad \mathrm{~B}(\mathrm{I}+1, \mathrm{~J}+\mathrm{I})=\mathrm{A}(\mathrm{I}-1, \mathrm{~J})+\mathrm{E}(\mathrm{I}, \mathrm{J}) S_{2} \\ 20 \quad \text { CONTINUE }\end{array}$

(a) Loop Nest with Non-vector Inner Loop

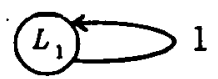

(b) DG for DOSPREAD Loop of (a)

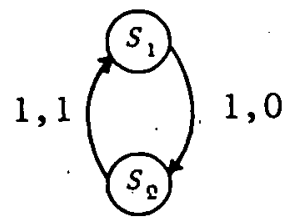

(c) DG for Nested Loop of (a)

Figure 18. Loop Splitting a Nested Loop 
10

DOSPREAD $40 \mathrm{I}=1, \mathrm{~N}$

DO $10 \mathrm{~J}=1,\left[\frac{M}{L}\right.$

$L_{1}^{1}$

$S_{1}$

CONTINUE

DO $L_{k} \mathrm{~J}=\left\lceil\frac{M}{L}\right\rceil+1,2^{*}\left\lceil\frac{M}{L}\right\rceil \quad: L_{1}^{k}$

$S_{1}$

CONTINUE

DO $\dot{L}_{l-1} \mathrm{~J}=(l=1)^{*}\left\lceil\frac{M}{L}\right\rceil+1, M \quad L_{1}^{l-1}$

$S_{1}$

CONTINUE

40 CONTINUE

(d) Loop Nest of (a) after Loop Splitting

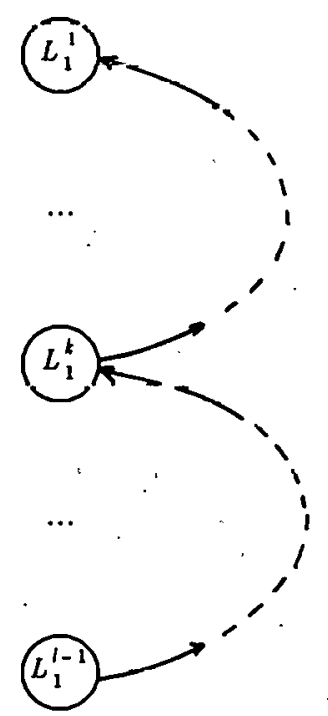

(e) The DG for the Split Loop of (d)

Figure 18. Continued 
Theorem 1 If of $f$ set sub-loops execute between the source and sink of a dependence, the dependence will be honored.

Proof By definition, a dependence will be honored if $\Delta_{\max }^{L}$ iterations execute between its source and sink, where $\Delta_{\max }^{L}$ is the maximum distance of any dependence on some loop L. Let. $I$ be the minimum number of iterations that executes between the source and sink for a given offset. Then

$$
\begin{aligned}
I & =\mid \text { off } f \text { set } \cdot \iota \mid \\
& \left.=\mid \imath \cdot\left(\mid \iota-\Delta_{\max }^{i}\right]-1\right) \mid \\
& \geq\left|\left(\iota-\Delta_{\max }^{L}\right)-\iota\right| \\
\geq & \left|\iota-\Delta_{\max }^{L}-\iota\right| \\
& \geq\left|-\Delta_{\max }^{L}\right|
\end{aligned}
$$

\begin{tabular}{|c|c|c|}
\hline direction & $i$ & offset \\
\hline$<\left(\Delta_{\max }^{J}>0\right)$ & $\begin{array}{l}\iota \leq \Delta_{\max }^{J} \\
\iota>\Delta_{\max }^{J}\end{array}$ & $\begin{array}{l}\Delta_{m a x}^{J} \leq \text { of fset }<0 \\
\text { offset }=0\end{array}$ \\
\hline$=\left(\Delta_{\max }^{J}=0\right)$ & all values & $o f f$ set $=0$ \\
\hline$>\left(\Delta_{\max }^{J}<0\right)$ & $\begin{array}{l}\iota \leq\left|\Delta_{\max }^{J}\right| \\
\iota>\left|\Delta_{\max }^{J}\right|\end{array}$ & $\begin{array}{l}1<\text { offset } \leq-\left|\Delta_{\max }^{J}\right| \\
\text { offset }=1\end{array}$ \\
\hline
\end{tabular}

Figure 19. Proof of Validity of OFFSET values

Figure 20. Values of Offset for Different Dependence Distances and $\iota$

the rows of an iteration space diagram into a single row. Figure 21 a gives a loop nest with ' $<$ ' 
dependences on both the $\mathrm{I}$ and $\mathrm{J}$ loops, and $\Delta_{\max }^{J}=3$. Figure $21 \mathrm{~b}$ gives the $\mathrm{DG}$ for the nested loop, and Figure 21c gives the iteration space diagram. Finally Figure 21d gives the IDG.

In Figure 22a the IDG of Figure 21d has been marked to show the range of sub-loops where $\iota=1$. All dependences to a particular sub-loop begin at an earlier sub-loop $\dot{L}_{k-2}$. That all

DOSPREAD $20 \mathrm{I}=1, \mathrm{~N}$
DO J $=101,20$
$\mathrm{~A}(\mathrm{I}+1, \mathrm{~J}+3)=\mathrm{B}(\mathrm{I}-1, \mathrm{~J})+\mathrm{C}(\mathrm{I}, \mathrm{J}) S_{1}$
$\mathrm{~B}(\mathrm{I}, \mathrm{J}+3)=\mathrm{D}(\mathrm{I}, \mathrm{J})+\mathrm{E}(\mathrm{I}, \mathrm{J}) \cdot S_{2}$
10
$20 \quad$ CONTINUE

(a) A Loop Nest

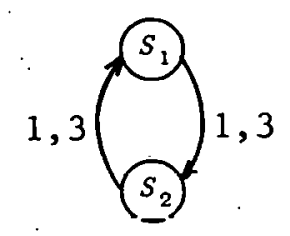

(b) DG for the J Loop of (a)

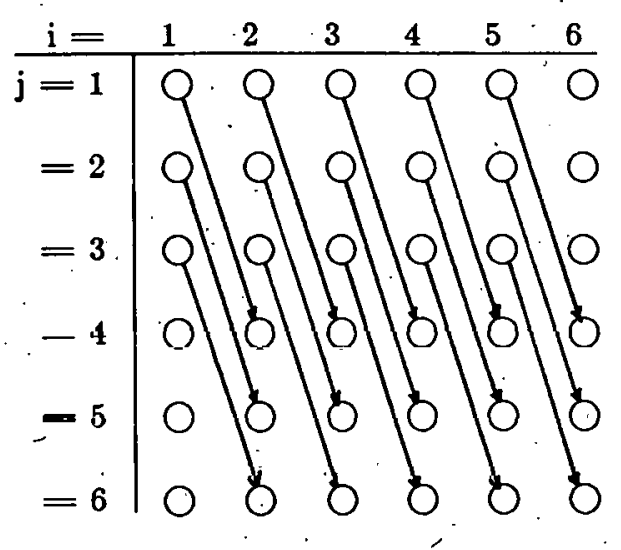

(c) Iteration Space for (a)

Figure 21. Conlinued 


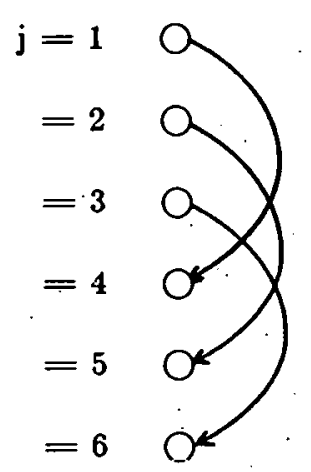

(d) IDG for (c)

Figure 21. Loop Nest Appropriate for Loop Splitting and its Associated IDG

dependences begin at an earlier sub-loop will always be true if $\iota<\left|\Delta_{m a x}^{J}\right|$, since the dependence must be from an earlier iteration and the. sub-loop is too small to contain both the source and the sink of the dependence.

In Figure 22b we can see the IDG with sub-loops marked where $\iota=4$. In this case, where $\iota>\left|\Delta_{\max }^{J}\right|$, a dependence whose sink is in some $L_{k}$ may also have its source in $L_{k}$. Therefore sub-loop $L_{k}$ cannot execute until sub-loop $L_{k}$ has finished in a previous iteration of the dospread loop. Synchronization accomplishing this occurs when of $f$ set $=0$. This remains true no matter how much larger $\iota$ becomes. Figure $22 \mathrm{c}$ shows the loop split with $\iota=4$. Figure $22 \mathrm{~d}$ shows the DG for the loop of Figure 22c, and Figure 22e shows the execution order of the iterations in the program.

In Figure 23a we have a loop nest where $\Delta_{\max }^{J}=0$. Figure 23b gives the IDG for the nested loop. Sub-loops with $\iota=2$ are shown on the IDG for the nested loop. Regardless of the size of $\iota$, sub-loops must always have dependences whose source and sink are within the sub-loop: Thus, sub-loop $L_{k}$ in some iteration of the dospread loop must always wait for sub-loop $L_{k}$ in soine previous iteration of the dospread loop to finish. Again, of $f$ set $=0$ accomplishes this. 
32

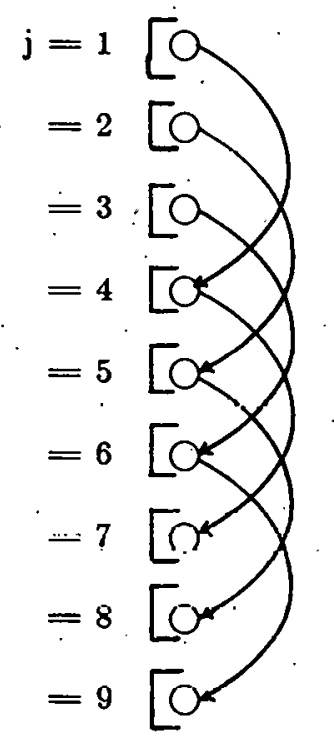

(a) IDG of Figure 21d Marked for $\iota=1$

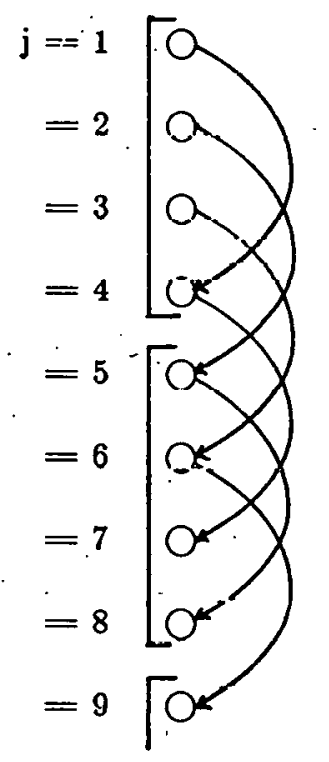

(b) IDG of Figure 21d Marked for $\iota=4$

Figure 22. Loop Splitting with ' $<$ ' Dependence 


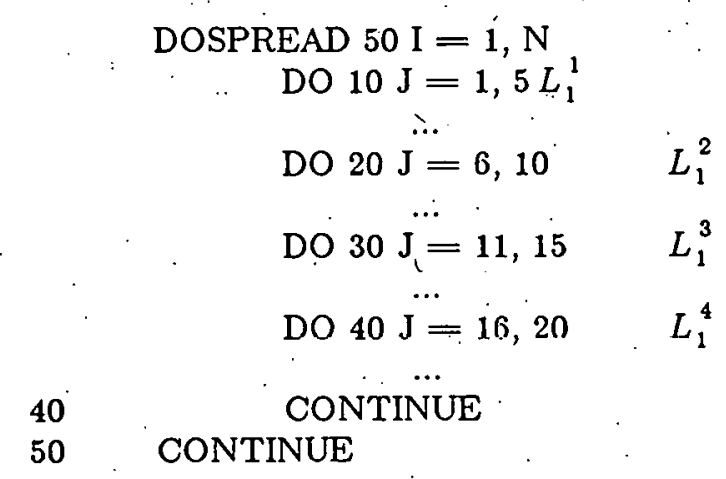

(c) Loop Nest after Splitting, with $\iota=4$

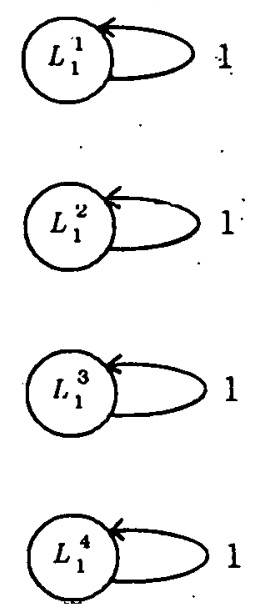

(d) The DG for the Split Loop of (c)

Figure 22. Continued 


\begin{tabular}{|c|c|c|c|c|}
\hline $\mathrm{i}=$ & 1 & 2 & 3 & 4 \\
\hline$t=1$ & $L_{1}^{1}$ & & & \\
\hline$\therefore=2$ & $L_{1}^{2}$ & $L_{1}^{1}$ & & \\
\hline$=3$ & $L_{1}^{3}$ & $L_{1}^{2}$ & $L_{1}^{1}$ & \\
\hline$=4$ & $L_{1}^{4}$ & $L_{1}^{3}$ & $\dot{L}_{1}^{2}$ & $L_{1}^{1}$ \\
\hline$=5$ & & $L_{1}^{4}$ & $L_{1}^{3}$ & $L_{1}^{2}$ \\
\hline$=6$ & & . & $L_{1}^{4}$ & $L_{1}^{3}$ \\
\hline$=7$ & & & & $\dot{I}_{1}^{4}$ \\
\hline
\end{tabular}

(e) Execution Order of the Sub-loops

Figure 22. Continued

Figure 23c shows the transformed program, Figure 23d its DG, and Figure 23e the execution order of the iterations.

Figure 24a shows a loop nest where the nested loop has $\Delta_{\max }^{J}=3$. Figure $24 \mathrm{~b}$ gives the IDG for the nested loop, showing sub-loops where $\iota=4$. When $\Delta_{\text {max }}^{J}>0$, some sources for dependences whose sinks are in the sub-loop will always be in a later sub-loop than the sink. Thus it is always true that of $f$ set $>0$. If $\iota \leq \Delta_{m a r}^{J}$, then no dependence can extend further than some sub-loop to the previous sub-loop. Therefore of $f$ set will equal 1 . If $\iota<\Delta_{\max }^{J}$, then a dependence will extend through several sub-loops, and of $f$ set will always be greater than 1: Figure 24c shows the transformed loop nest, Figure 24d the DG for the loop, and Figure 24e shows the execution order of the iterations.

Figure 25 summarizes the rules for handling nested loops.

\subsubsection{Splitting Loops with '<' and '=' Direction Components}

If a loop contains only dependences with ' $<$ ' and ' $=$ ' direction components, the software necessary to split these loops is simpler than in the more general case. For reason for this can be seen by examining the table of Figure 20. If the nested loop contains only ' $<$ ' and ' $=$ ' direction 


DOSPREAD $20 \mathrm{I}=1, \mathrm{~N}$
DO $10 \mathrm{~J}=1,8$
$\mathrm{~A}(\mathrm{I}, \mathrm{J})=\mathrm{B}(\mathrm{I}, \mathrm{J})+\mathrm{C}(\mathrm{I}, \mathrm{J})$
$\mathrm{D}(\mathrm{I}, \mathrm{J})=\mathrm{A}(\mathrm{I}-2, \mathrm{~J})+\mathrm{E}(\mathrm{I}, \mathrm{J})$

(a) Program with '=' Dependence on the Nested Loop

$$
\begin{array}{ll}
\mathrm{j}=1 & \leftrightarrow 2 \\
=2 & \leftrightarrow 2 \\
=3 & \leftrightarrow 2 \\
=4 & \leftrightarrow 2
\end{array}
$$

(b) IDG for (a)

$$
\begin{gathered}
\text { DOSPREAD } 50 \mathrm{I}=1, \mathrm{~N} \\
\text { DO } 10 \mathrm{~J}=1,2 L_{1}^{1} \\
\cdots \\
\text { DO } 20 \mathrm{~J}=3,4 L_{1}^{2} \\
\ldots \\
\text { DO } 30 \mathrm{~J}=5,6 L_{1}^{3} \\
\text { DO } 40 \mathrm{~J}=7,8 L_{1}^{4}
\end{gathered}
$$

50 CONTINUE

(c) Loop Nest of (a) after Loop-splitting

Figure 23. Loop Splitting with ' Dependencec 

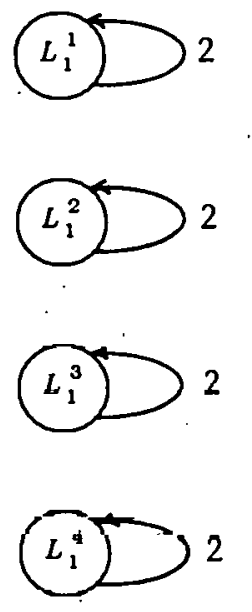

(d) The DG for the Split Loop of (c)

\begin{tabular}{c|cccccc}
$\mathrm{i}=$ & 1 & 2 & 3 & 4 & 5 & 6 \\
\hline $\mathrm{t}=1$ & $L_{1}^{1}$ & $L_{1}^{1}$ & & & & \\
$\mathrm{t}=3$ & $L_{1}^{2}$ & $L_{1}^{2}$ & $L_{1}^{1}$ & $L_{1}^{1}$ & & \\
$\mathrm{t}=4$ & $L_{1}^{3}$ & $L_{1}^{3}$ & $L_{1}^{2}$ & $L_{1}^{2}$ & $L_{1}^{1}$ & $L_{1}^{1}$ \\
$\mathrm{t}=5$ & $L_{1}^{4}$ & $L_{1}^{4}$ & $L_{1}^{3}$ & $L_{1}^{3}$ & $L_{1}^{2}$ & $L_{1}^{2}$ \\
$\mathrm{t}=6$ & & & $L_{1}^{4}$ & $L_{1}^{4}$ & $L_{1}^{3}$ & $L_{1}^{3}$ \\
$\mathrm{t}=7$ & & & & & $L_{1}^{4}$ & $L_{1}^{4}$.
\end{tabular}

(e) Execution Order of Split Loops

Figure 23. Continued 


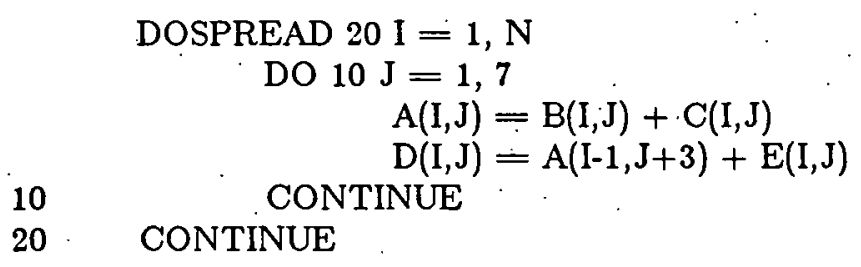

(a) Loop Nest with '>' Dependences

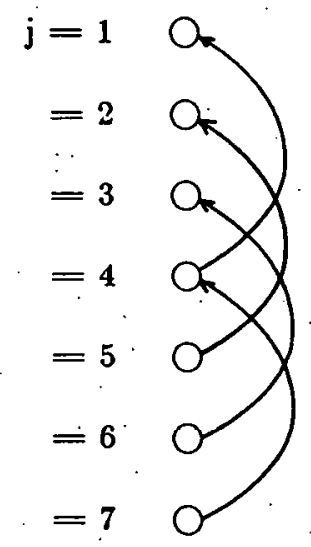

(b) IDG for (a)

$$
\begin{array}{r}
\text { DOSPREAD } 50 \mathrm{I}=1, \mathrm{~N} \\
\text { DO } 10 \mathrm{~J}=1,2 L_{1}^{1} \\
\cdots \\
\text { DO } 20 \mathrm{~J}=2,4 L_{1}^{2} \\
\ldots \\
\text { DO } 30 \mathrm{~J}=5,6 L_{1}^{3} \\
\text { DO } 10 \mathrm{~J}=7,7 L_{1}^{4}
\end{array}
$$

50 CONTINUE

(c) Loop Nest of (a) after Loop Splitting

Figure 24. Loop Splitting with ' $>$ ' Dependences 


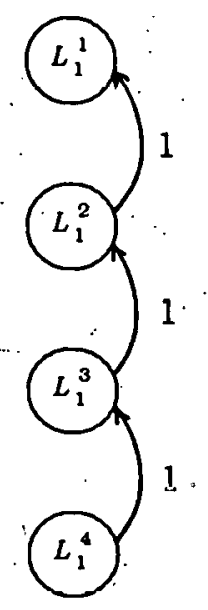

(d) The DG for the Split Loop of (d)

\begin{tabular}{|c|c|c|c|c|}
\hline $\mathrm{i}=$ & 1 & 2 & 3 & 4 \\
\hline $\mathrm{t}=1$ & $L_{1}^{1}$ & & & \\
\hline-2 & $L_{1}^{2}$ & & & \\
\hline$=3$ & $L_{1}^{3}$ & $L_{1}^{1}$ & & \\
\hline$=4$ & $L_{1}^{4}$ & $L_{1}^{2}$ & & \\
\hline$=5$ & & $L_{1}^{3}$ & $L_{1}^{\prime}$ & \\
\hline$=6$ & & $L_{1}^{4}$ & $L_{1}^{?}$ & \\
\hline$=7$ & & & $L_{1}^{3}$ & $L_{1}^{1}$ \\
\hline$=8$ & & & $L_{1}^{4}$ & $L_{1}^{2}$ \\
\hline$=9$ & & & & $L_{1}^{3}$ \\
\hline$=10$ & & & & $L_{1}^{4}$ \\
\hline
\end{tabular}

(e) Execution Order of the Sub-loops

Figure 24. Continued 
$D$ - a set of all dependences in the DG.

$L$ - the dospread loop being synchronized.

$L_{x b}$ - the upper bound of the loop for which the DG built.

$R$ - the number of synchronization registers.

$k$ - the minimum number of iterations in a loop that can be efficiently

executed in light of startup overheads, etc.

Distance $(\delta)$ returns the distance of a dependence $\delta$, and is defined as: return the actual maximum dependence distance, if determinable. Otherwise,

if the direction of $\delta$ is ' $<$ ' then

$$
\text { return }-1
$$

if the direction of $\delta$ is ' $=$ ' or ' $<=$ ' then return 0

if the direction of $\delta$ is ' $>$ ', ' $=>{ }^{\prime}$, or ' $<=>$ ' then return $L_{t b}$.

foreach dependence $\delta \in D$ do

if $\delta$ is immediately nested in a vector loop then fully distribute the vector loop.

foreach dependence $\delta \in D$ do

$L_{\theta o}$ is the outermost loop containing the source that is nested within the spread loop, or is undefined otherwise.

$L_{e i}$ is the outermost loop containing the source that is nested within the spread loop, or is undefined otherwise.

if $L_{s o}=L_{o i} \neq$ undefined then

find $\Delta_{\min }^{\text {spread }}$, the minimum dependence distance of

any dependence completely within $L$.

remove $\delta$ and all other $\delta^{\prime}$ completely within $L$ from $D$

build a DG for $L_{B 0}$, including all dependences regardless of direction.

find $\Delta_{m a x}^{L}$, the maximum distance of a dependence in $L_{s o}$.

L. $:=\max \left(k,\left[L_{u b} / R\right]\right)$

computc of foct as given in the text

split the loop into $\left\lceil\frac{L_{a b}}{\iota}\right]$ sub-loops, numbered lexically from 1 to $\mathrm{n}$.

foreach dependence $\delta " \in D$ do

if source $\left(\delta^{\prime \prime}\right)=\mathrm{DO}$ of $L$ or $\sin k\left(\delta^{\prime \prime}\right)=$ CONTINUE of $L$ then

foreach sub-loop $!$ do replicate the dependence to or from each $L_{i}, 1 \leq i \leq n$ place a dependence from the end of the sub-loop to the beginning of sub-loop $j, j=l+$ of $f$ set. If $j<1$ do not add the dependence. Mark the dependence as having a distance of $\Delta_{\min }^{\text {spread }}$

Figure 25. Algorithm for Nested Loops 
components, then we can assume that the value of $\ell$ is always greater than the value of $\Delta_{\max }^{J}$. This can be done because this assumption always leads to the most conservative synchronization for this case. Therefore, for any $\iota$, of $f$ set $=0$. If ' $>$ ' direction components are present, then the most conservative synchronization requires assuming $\Delta_{\text {max }}^{J}$ equals the upper bound of the nested $\mathrm{J}$ loop. This in turn leads to the nested $J$ loop being run completely serially, with synchronization to insure the dependence is filled before the loop, and synchronization to show that the depcndence is filled after the loop. This means that, to synchronize nested loops with '>' direction components, we need an upper bound on the value of $\Delta_{\max }^{J}$ less than the upper bound of the $J$ loop in order to salvage some parallelism, whereas in the latter case we need only know the direction.

\subsubsection{Removing Extraneous Dependences Introduced in L̀oop Splitting}

It is possible that loop splitting will cause extraneous dependence to be introduced into the DG. These dependences result from a dependence source or sink being moved out of a loop, as in Sections 2.2.1 and 2.2.2, and the loop being subsequently split. As the loop is split, the dependences attached to the DU and CONTINUE statements of the loop are replicated, and allached to the DO and CONTINUE statements of each sub-loop. Figure 26a gives a program, Figure 26h its DG, and Figure 26c the DG after splitting the inner loop into four sub-loops. Leaving these dependences in the $\bar{D} G$ will not cause incorrect synchrontzation to be generaled, bul il may cause unnecessary synchronization to be generated. And in the case of the extra dependences being LBDs, the parallelism gained by loop splitting can be eliminated by the extra dependences. Therefore the extra dependences should be removed if possible.

To do this, it is necessary only to retrieve the source and sinks from the dependence arcs, and repeat dependence testing on these statements. If a dependence is not present, then the corresponding arc can be eliminated. Figure 26d shows the DG of Figure 26c after performing additional data dependence testing. 


$$
\begin{array}{cc}
\text { DO } 20 \mathrm{I}=1, \mathrm{~N} \\
\mathrm{~B}(\mathrm{I})=\mathrm{B}(\mathrm{I})+\mathrm{A}(\mathrm{I}-1, \mathrm{I}) \\
\mathrm{DO} 10 \mathrm{~J}=1, \mathrm{~N} \\
\quad \mathrm{~A}(\mathrm{I}, \mathrm{J})=\mathrm{C}(\mathrm{I}, \mathrm{J})+\mathrm{D}(\mathrm{I}, \mathrm{J}) \\
\mathrm{E}(\mathrm{I}, \mathrm{J})=\mathrm{A}(\mathrm{I}-1, \mathrm{~J})+\mathrm{F}(\mathrm{I}, \mathrm{J}) \\
10 \quad \\
\quad \text { CONTINUE } \\
20 \quad
\end{array}
$$

(a) A Loop Nest

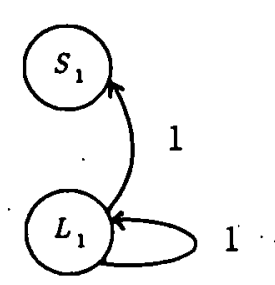

(b) The DG for (a).

Figure 26. Extraneous Dependence Removal Example 


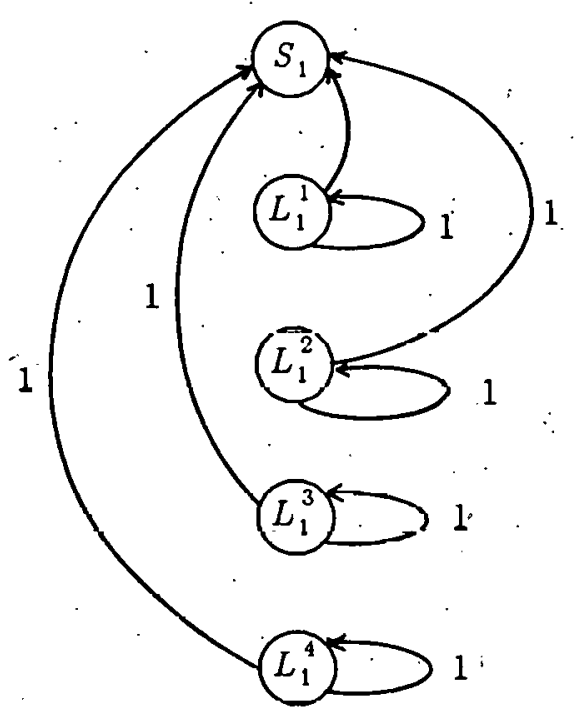

(c) The DG after Splitting the Inner Loop into Four Sub-loops
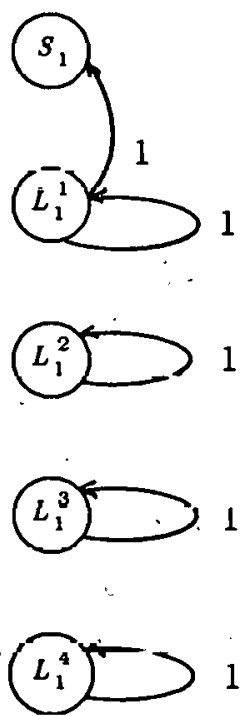

(d) The DG after Removing Extraneous Dependences

Figure 26. Continued 


\section{CHAPTER 3}

\section{GENERATION OF SYNCHRONIZATION INSTRUCTIONS}

At this point a DG has been constructed in the previous chapters that accurately reflects the dependence relations among statements in the program that must be honored. This chapter uses this information to add synchronization statements to the source program. We cover two major topics in doing this. The first is the definition of two sets of synchronization instructions, and how they can be used to synchronize loops in the absence of any control branches in the source loop. The second is the generation of synchronization in the presence of control branches in the source loop.

\subsection{Synchronization Instructions}

We use two different sets of synchronization instructions in this thesis. The first set consists of the test and set instructions, the second set of the test and testset instructions. The test instructions in the two sets are different, but this will not cause any problems since the two instruction sets will never be intermixed in a program. Both instruction sets contain the quit instruction. The semantics of synchronization instructions are defined only within a dospread loop. The relative advantages of the two instruction sets are covered in section 3.3 .

\subsubsection{SET and TEST Instructions}

The test and set instructions operate on synchronization registers that are accessed as bit vectors. The registers are long enough so that there is one bit per iteration of the loop. If the execution order of the iterations of a loop is constrained so that the lowest running iteration number is never $k$ more than the highest running iteration number, then the register needs to only have $k$ bits. This is not too difficult to enforce if loops are scheduled at run time, i.e are 
not scheduled by blocking. When the dospread loop begins running, all bits are assumed to be initialized to zero. We will refer to bit $i$ of register $r$ as $r[i]$.

The set instruction has the syntax set $(r)$, where $r$ is a synchronization register. If a set instruction executes in some iteration $i$, it performs the assignment $r[i]:=1$.

The test instruction has the syntax $\operatorname{test}(r) \Delta$, where $r$ is a synchronization register and $\Delta$ is a dependence distance. If $\operatorname{test}(r) \Delta$ executes on iteration $i$, then it busy wails for $r[i-\Delta]$ to be equal to one. Figure 27 summarizes the actlons of these instructions.

The set instruction signals the completion of an action, in particular the execution of a dependence source. The test waits until the completion of that action before starting another action such as execution of a dependence sink.

\subsubsection{TEST and TESTSET Instructions}

The test and tcstset instructions operation on a synchronization register that can hold an integer. The register should be able to hold an integer as large as the loop upper bound. 'l'he register is set to zero when the concurrent loop begins execution.

The testset instruction has the syntax testset( $r)$. It first checks to see if a testset( $r)$ instruction has been executed in the previous iteration. This it does by checking if the value of

$$
r[i]:=1
$$

(a) Semantics of $\operatorname{set}(r)$ executing in iteration $i$.

$$
l: \quad \text { if } i-\Delta>0 \text { then } \quad \text { if } r[i-\Delta]=0 \text { then goto } l
$$

(b) Semantics of test $(r) \Delta$ executing in iteration $i$.

Figure 27. Semantics of the set and test Instructions 
$r=i-1$, where $i$ is the iteration number (not the value of the loop index) of the testset executing. If so, it increments $r$ making it equal to $i$, otherwise it waits. Figure 28a summarizes this.

The test instruction has the syntax test $(r) \Delta$, where $\Delta$ is the dependence distance. A test instruction in iteration $i$ busy waits on $r$ until $r=i-\Delta$, i.e. until a testset $(r)$ is executed in iteration $i-\Delta$. Figure $28 \mathrm{~b}$ summarizes the semantics of the test instruction.

\subsubsection{The QUIT Instruction}

The syntax of the quit instructions is simply quit. Its purpose is to halt execution of a loop before all iterations of the loop have executed, and is used in this thesis only to terminate dospread loops when exited by an exit-if. When a guit statement is executed the parallel loop is terminated immediately on all processors executing the loop.

\subsection{Synchronizing Within Blocks of Assignment Statements (BAS)}

Synchronizing a dependence is easiest if no control branches occur between the source and sink of the dependence. In this section we deal with loops whose bodies are blocks of assignment

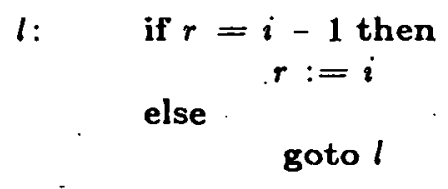

(a) Semantics of testset ( $r$ ) executing in iteration $i$.

$$
\begin{aligned}
& \text { if } i-\Delta>0 \text { then } \\
& \text { if } r<i-\Delta \text { then goto } l
\end{aligned}
$$

(b) Scmantics of $t \cot (r) \Delta$ in itcration $i$.

Figure 28. Semantics of the testset and test Instructions 
statements. The principles outlined in this section. also apply. if control branches occur, but additional actions need to be taken, as discussed later in this chapter. We show how to synchronize programs using both test and set, and test and testset instructions.

\subsubsection{Synchronizing LFDs and LBDs Using TEST and SET}

Consider the program of Figure 29a, and the DG of Figure 29b. There exists a LFD of distance 2 from $S_{1}$ to $S_{2}$ on $A$. To insure that the dependence is honored, $S_{2}$, on any iteration $i>2$, must execute after $S_{1}$; on iteration $i-2$, execules. This means a set instruction should be placed after $S_{1}$, and a test instruction, with $\Delta=2$; before $S_{2}$. The synchronized program is shown 'in Figure 29c.

10

$$
\begin{aligned}
& \text { DOSPREAD } 10 \mathrm{I}=1, \mathrm{~N}
\end{aligned}
$$

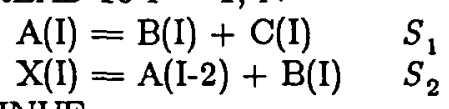

$$
\begin{aligned}
& \text { CONTINUE }
\end{aligned}
$$

(a) Program with LFW

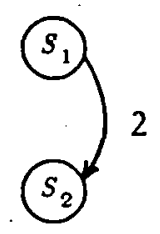

(b) DG for the Program of (a)

10 CONTINUE

$$
\begin{aligned}
\text { DOSPREAD } 10 \mathrm{I}=1, \mathrm{~N} \\
\\
\mathrm{~A}(\mathrm{I})=\mathrm{B}(\mathrm{I})+\mathrm{C}(\mathrm{I}) \\
\operatorname{SET}(1) \\
\text { TEST }(1) 2 \\
\mathrm{X}(\mathrm{I})=\mathrm{A}(\mathrm{I}-2)+\mathrm{B}(\mathrm{I}) \quad S_{1}
\end{aligned}
$$

(c) Program with Test and Set Instructions Added

Figure 29. Program and DG with LFD 
The program and DG of Figure $30 \mathrm{a}$ and Figure $30 \mathrm{~b}$, respectively, show a LBD. The goal is the same as with a LFD, namely to notify the dependence sink at $\dot{S}_{1}$ when the source at $S_{2}$ has executed. Again, this is done by placing a set after the source and a test before the sink of the dependence. The synchronized program is shown in Figure $30 \mathrm{c}$.

In general, with a dependence having distance $\Delta$ from $S_{i}$ to $S_{j}$, set $\left(r_{\varepsilon}\right)$-placed after the source of the dependence and test $\left(r_{8}\right) \Delta$ placed before the sink of the dependence will force the dependence to be honored.

DOSPREAD $10 \mathrm{I}=1, \mathrm{~N}$

$$
\begin{aligned}
& \dddot{\mathrm{B}}(\mathrm{I})=\mathrm{A}(\mathrm{I})+\mathrm{C}(\mathrm{I}) \quad: S_{1} \\
& \mathrm{~A}(\mathrm{I}+2)=\mathrm{B}(\mathrm{I})+\mathrm{C}(\mathrm{I}) \quad S_{2}
\end{aligned}
$$

10 CONTINUE

(a) Program with LBD

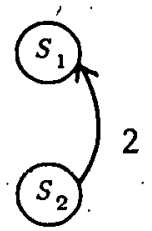

(b) DG for the Program of (a)

DOSPREAD $10 \mathrm{I}=1, \mathrm{~N}$

TEST(1) 2

$\mathrm{B}(\mathrm{I})=\mathrm{A}(\mathrm{I})+\mathrm{C}(\mathrm{I}) \quad S_{1}$

$\mathrm{A}(\mathrm{I}+2)=\mathrm{B}(\mathrm{I})+\mathrm{C}(\mathrm{I}) \quad S_{2}$

SET(1)

$10 \cdot$ CONTINUE

(c) Program with Test and Set Instructions Added

Figure 30. Program and DG with LBD 
Figure 31 summarizes these rules.

\subsubsection{Synchronizing LFDs and LBDs Using TEST and TESTSET}

The goals of synchronizing using test and testset are the same as with test and set. With LFDs, fulfilling this goal is easier using the testset instruction. Again consider the program of Figure 29a. To synchronize this program we need only to place a testset between $S_{1}$ and $S_{2}$. This is shown in Figure 32. $S_{2}$ in iteration $i$ cannot execute before the testset in iteration $i$, since the testset precedes $S_{2}$. But the testset of iteration $i$ cannot execute before the testset of iteration $i-1$, which cannot execute before the lestset of iteration of $i-2$. But the testset of $i-2$ cannot execute until $S_{1}$ in $i-2$ has finished, since $S_{1}$ precedes the testset. Thus $S_{1}$ in iteration $i-2$ must execute before $S_{2}$ in iteration $i$, and the dependence is satisfied. Therefore LFDs can be synchronized by putting a testset between the source and the sink of the LFD.

If $\Delta$ for a dependence being synchronized is not constant, then the test instruction should be given the minimum value of $\Delta, \Delta_{\min }$ : As can be seen from the algorithms of Chaptcr 4 , this

foreach dependence $\delta=\left(\dot{S}_{a 0}, S_{a i}\right)$, with distance $\Delta$ do afteir $S_{o s}$ place the stalemenl $\operatorname{sel}\left(r_{i}\right)$

before $S_{e i}$ place the statement test $\left(r_{i}\right) \Delta$

Figure 31. Placing Test and Set Instructions in BAS

$\begin{array}{ccc}\text { DOSPREAD } 10 \overline{\mathrm{I}}=1, \mathrm{~N} \\ \mathrm{~A}(\mathrm{I})=\mathrm{I}(\mathrm{I})+\mathrm{C}(\mathrm{I}), & s_{1} \\ & \text { TESTSET(1) } \\ \mathrm{X}(\mathrm{I})=\mathrm{A}(\mathrm{I}-2)+\mathrm{B}(\mathrm{I}) & s_{2} \\ 10 \quad \text { CONTINUE } & \end{array}$

Figure 32. Program with LF'U Synchronized using TEST 
is the same as synchronizing for each value of $\Delta$ and then eliminating the redundant dependence arcs.

LBDs are synchronized as before with test and set, except now testset is used instead of set to signal test. Figure 33 shows the program of Figure $30 \mathrm{a}$ synchronized using test and testset .

Figure 34 summarizes these rules.

\subsection{Advantages and Disadvantages of the Instruction Sets}

Now that we know how the instructions are used to synchronize dependences, it is possible to examine the relative advantages of the two instruction sets. The most obvious difference is

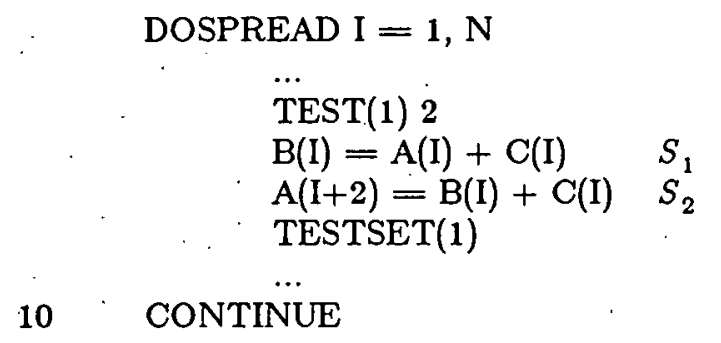

Figure 33. Program with LBD Synchronized using TEST and TESTSET

foreach dependence $\delta=\left(S_{s 0}, S_{s i}\right)$ with distance $\Delta$ do if $s o<s i$ then place the instruction testset $\left(r_{i}\right)$ after $S_{\theta 0}$ and before $S_{\theta i}$.

else place the instruction testset $\left(r_{i}\right)$ after $S_{80}$ place the instruction te.st $\left(r_{i}\right) \Delta$ before $S_{\theta i}$

Figure 34. Placement of test and testset Instructions in BAS' 
the amount of storage needed for a synchronization register. With a bit needed for each iteration, test and set instructions will need 4096 bytes of storage to synchronize a loop with an upper bound of 32767 , whereas the test and testset instruction will need only two bytes. As has been stated, though, by restraining the execution of iterations, the storage requirements for test and set can be eased.

More importantly, using test and $s e l$, il is pussible to execute a forall loop completely in parallel. Using test and testset requires that any loop requiringi synchronization have a serial component. Figure 35a gives a loop that has been synchronized using test and set instructions. Figure $35 \mathrm{~b}$ gives an optimal execution order of the statements. At time $t=2$ every iteration $i$. will set bit $i$ of $r$. At time $t=3$ every iteration $i$ will read bit, $i-1$ of $r$. Since each processor is accessing a different element of $r$ at each time step, the loop can execute completely in parallel. Of course, if some processors are delayed, the loop may not execute completely in parallel, but it is still theoretically possible for it to do so.

Using the testset instruction, this is not possible. Figure 36a gives the same loop as before, except now synchronized using a testset instruction. Figure $36 \mathrm{~b}$ gives the optimal execution order of the statements in the loop. Note that now six time steps are needed to execute lite lour lterations of the loop. This is because the testset of iteration two must wait for the testset of iteration 1 to finish before it executes, and cannot execute in parallel with the synchronization instructions of other iterations as in the previous example. Therefore the savings of space in the synchronization registers is paid for in execution time.

Another difference in the two instructions will become apparent when we discuss dependence arc elimination in Chapter 4. The testset instruction is more powerful than what is needed to simply synchronize many dependences. The additional power allows us to potentially use fewer synchronization instructions with test and testset instructions than with test and set instructions. 


$\begin{array}{ccc} & \text { DOSPREAD } 10 \mathrm{I}=1,4 \\ & \mathrm{~A}(\mathrm{I})=\mathrm{B}(\mathrm{I})+\mathrm{C}(\mathrm{I}) & S_{1} \\ \operatorname{SET}(r) & S_{s} \\ & \operatorname{TEST}(r) 1 & S_{t} \\ & \mathrm{D}(\mathrm{I})=\mathrm{E}(\mathrm{I})+\mathrm{A}(\mathrm{I}-1) & S_{4} \\ 10 \quad \text { CONTINUE }\end{array}$

(a) A Loop Synchronized Using TEST and SET Instructions

\begin{tabular}{c|cccc}
$\mathrm{i}=$ & 1 & 2 & 3 & $\mathbf{4}$ \\
\hline $\mathrm{t}=1$ & $S_{1}$ & $S_{1}$ & $S_{1}$ & $S_{1}$ \\
$=2$ & $S_{s}$ & $S_{s}$ & $S_{s}$ & $S_{g}$ \\
$=3$ & $S_{t}$ & $S_{\mathrm{t}}$ & $S_{\mathrm{t}}$ & $S_{\mathrm{t}}$ \\
$=4$ & $S_{2}$ & $S_{2}$ & $S_{2}$ & $S_{2}$
\end{tabular}

(b) Optimal Execution of (a)

Figure 35. Optimal Execution of a Loop using TEST and SET Instructions

10

$\begin{array}{cc}\text { DOSPREAD } 10 \mathrm{I}=1,4 \\ \mathrm{~A}(\mathrm{I})=\mathrm{B}(\mathrm{I})+\mathrm{C}(\mathrm{I}) & S_{1} \\ \mathrm{TESTSET}(r) & T S \\ \mathrm{D}(\mathrm{I})=\mathrm{E}(\mathrm{I})+\mathrm{A}(\mathrm{I}-1) & S_{2} \\ \text { CONTINUE }\end{array}$

(a) A Loop Synchronized Using TEST and TESTSET Instructions.

\begin{tabular}{r|cccc}
$\mathrm{i}=$ & 1 & 2 & 3 & 4 \\
\hline $\mathrm{t}=1$ & $S_{1}$ & $S_{1}$ & $S_{1}$ & $S_{1}$ \\
$=2$ & $T S$ & & & \\
$=3$ & $S_{2}$ & $T S$ & & \\
$=4$ & & $S_{2}$ & $T S$ & \\
$=5$ & & & $S_{2}$ & $T S$ \\
$=6$ & & & & $S_{2}$
\end{tabular}

(b) Optimal Execution of (a)

Figure 36. Optimal Execution Using TEST and TESTSET Instructions 


\subsection{Loop Modifications for Exit-ifs}

In Chapter 2 the DG was modified to reflect the presence of exit-ifs: This modification insured that a loop would only begin executing if the previous loop did not take the exit branch of an IF statement. If the exit branch of an IF statement was taken, processors executing later iterations of the loop will deadlock while waiting for a signal that they can begin their iterations unless a quit instruction is executed. The quit cannot be immediately executed, however, as this might halt the execution of an iteration previous to the exiting iteration. This section shows how the loop can be modified so that these problems are overcome.

The solution to the above problem requires both adding synchronization instructions and additional FORTR NN statements. To insure all iterations less than the terminating iteration have completed we will place set(0) or testset $(0)$ at the end of the loop body. All branches within the loop to the CONTINUE statement at the end of the loop will instead branch to the set or testset instruction. Therefore, each time a loop iteration ends, synchronization register 0 will be set.

To terminate the dospread loop the processor exiting the loop needs to exocute a quit instruction when all previous iterations have finished. This is done by adding a block of code to the end of the loop for each exit-if target in the loop. If an exit-if target is $T$, then at the bottom of the loop the following code sequence is added:

\section{$T^{\prime} \operatorname{TEST}(0) 1$ \\ QUIT GOTO T}

The test instruction makes sure that all iterations previous to this iteration have finished. If they have, the quit instruction is executed, whirh releases all processors from cxccuting this loop, and allows them to continue to execute other code. Finally, the GOTO instruction causes the terminating processor to branch to the target of the exit-if. 
To signal the termination of a loop a statement $\operatorname{set}(0)$ is added immediately before the blocks just added that terminates and exits the loop. To prevent the loop from falling into the just added blocks a GOTO to the CONTINUE statement is placed immediately after the set $(0)$ statement. If $L$ is the label of the CONTINUE statement, then this appears as:

$\operatorname{SET}(0)$

GOTO L

Figure $37 \mathrm{a}$ shows the program of Figure $15 \mathrm{a}$, and Figure $37 \mathrm{~b}$ the program with all the changes necessary to synchronize the exit-if.

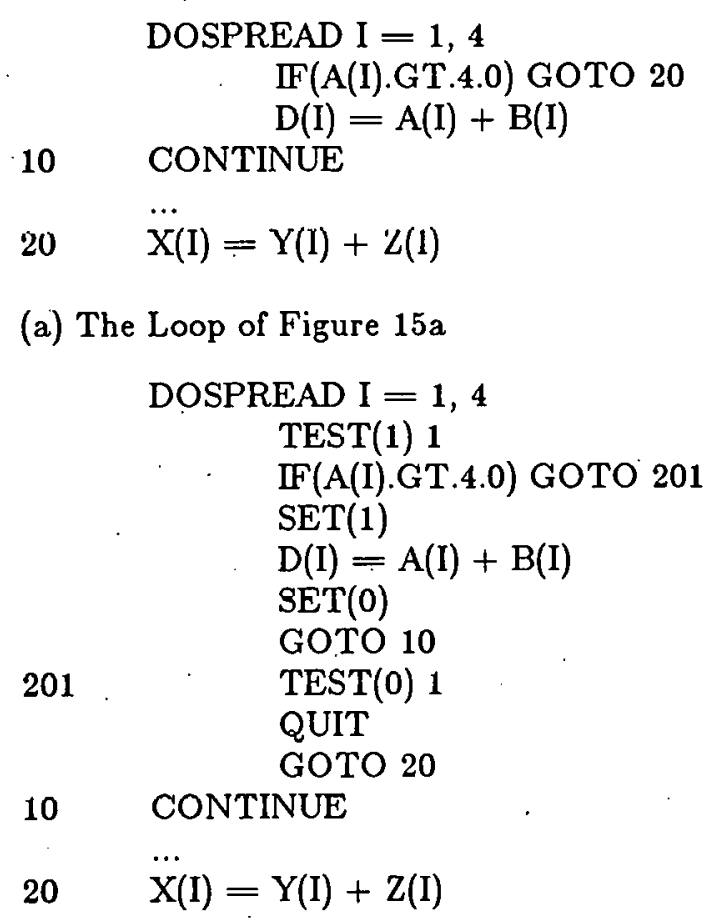

(a) The Loop of Figure 15a

(b) The Loop of (a) With Syncluvinizaliun for the Exil-if Added

Figure 37. Exit-if Example: Completely Synchronized Exit-if 
Figure 38 summarizes the rules for synchronizing exit-ifs.

\subsection{Adding Instructions in Loops with Complex Flow of Control}

The previous discussion covered the simple case of adding instructions to:loops whose bodies are BASes. In the more general case the loop body is not a BAS, but instead contains arbitrary forward branches within the loop. We.now show how to handle this more complex case. A graph is first constructed that represents the flow of control within the loop body. Then, using this graph and the DG constructed previously, synchronization instructions are addcd to the loop.

$T$ is the set of targets of exit-ifs

$S_{c}$ is the CONTINUE statement at tine end of the spread loop.

$S_{b}$ is a GOTO to $S_{c}$.

$S_{1}$ is the first statement in the loop.

if exit-ifs exist then

place $S_{b}$ immediately before $S_{c}$.

place the statement set $(0)$ (or testsel( $\left.0^{\prime}\right)$ ) immediately before $S_{b}^{\prime}$.

foreach. exit-if $E$ do add the target of $E$ to $T$.

foreach $t \in T$ do

after $S_{b}$ place the statements

$\begin{array}{ll}\mathrm{t}^{\prime} \quad & \text { test }(0) 1 \\ & \text { quit } \\ & \text { GOTO } t\end{array}$

foreach exit-if with target $t$ do change $t$ to t'.

Figure 38. Exit-if Algorithm 


\subsubsection{The Flow of Control Graph (FCG)}

This section gives the algorithms for constructing the two flow of control graphs (FCG) needed to add synchronization instructions to the FORTRAN program and for eliminating dependences.

The FCG is represented as a boolean matrix, $\operatorname{reach}[i, j]$, where reach $[i, j]$ is true if and only if block $i$ can reach block $j$, without going through any other blocks. A block is similar to a basic block in [AhUl77] except that branches to targets outside the loop, nested DO statements, statements whose labels are the targets only of nested DO statements, and statements that terminate execution of the program unit (RETURN, STOP) are ignored. The first three are ignored because they have been dealt with in Chapter 2, and the DG already reflects their presence. The last are ignored because synchronization is moot if the program unit is halted. Figure 39 gives the algorithm for building the FCG for the body of a loop. It is again noted that the FCG must be acyclic since backward branches are not allowed.

The first and last statements in a block are held by block_limits. Block」imits[b].first gives the first statement in a block, and block」imits[b].last gives the last statement in a block. The block that a statement is in is contained in stmt_to_block. Stmt_to_block $[s]$ gives the block that encloses statement $s$. Both of these data structures are computed at the same time the FCG is. A second FCG, reach", is also needed. Reach ${ }^{*}$ is the transitive closure of reach. $\operatorname{Reach}^{*}(i, j)$ is true if block $i$ can reach block $j$.

A second FCG, built at the statement level, is used in Chapter 4. This graph, represented by the boolean matrix reach_stmt, is constructed using the data structures built during the construction of reach. Figure 40 gives the algorithm for building this.

\subsubsection{Adding Statements using the Flow of Control Graph (FCG)}

In this section we use the DG built in the previous chapters, and the FCG built in the previous section to add synchronization statements to a loop. Set and testset instructions are 
first is the first statement in the loop

last is the last statement in the loop

branch $(s)=$ true if statement $s$ is a branch not handled in Chapter 2.

label $(s)=$ true if statement $s$ is labeled and is the target of a branch

$/^{*}$ build block_limits */

$b:=1$

block」imits $[b]$. first $:=$ first

for $s:=$ first to last -1 do

if $\operatorname{branch}(s)$ or label $(s+1)$ then

block」Jimits [b]. last $:=s$

block」imits $[b+1]$. first $:=s+1$

$b:=b+1$

block」imits $[b]$. last $:=$ last

$j^{*}$ build stmt_to_block *j

for $i:=1$ to $b$ do

for $j:=$ block」limits $[i]$.first to block_limits $[i]$.last do stmt_to_block $[j]:=i$

$/^{*}$ build reach */

reach $:=$ false

for $i:=1$ to $b$ - 1 do

if block_limits [i].last is a branch then

foreach non exit-if target $t$ in the branch do reach $[i$, stmt.tn_hlor.k $[t]]:=$ true.

Figure 39. Algorithm for Building a FCG, Block_limits and Stmt_to_block 
\#blocks = number of blocks in reach

$N_{f}:=$ block」limits (1). first c

$N_{l}:=$ block」limits (\#blocks).last

$/^{*}$ build a statement level flow graph */

stmi_reach $:=$ false

for $i:=1$ to \#blocks do

$/^{*}$ add arcs from non-branching statements */

for $j:=$ block_imils [i]. first to block_limits [i].last -1 do

stmt_reach $[j, j+1]:=$ true

${ }^{*}$ add arcs from possible branching statements * $/$

for $j:=1$ to \#blocks - 1 do

if reach $[i, j]=$ true then

stmt_reach [block」limits $[i]$.last, block」limits $[j]$. first $]:=$ true

Figure 40. Building a Statement Level Control Flow Graph

identical for the purposes of this section. Therefore we will only refer to the set instruction.

Both test instructions are also equivalent for the purposes of this section.

\subsubsection{Adding TEST Instructions}

Adding test instructions is trivial. The test instruction is placed immediately before the source of the dependence it is synchronizing. The only flow of control consideration is if the dependence source has a label. If so, the label must be moved to the test instruction so that it is not jumped over by the branch. The rules for this are given in Figure 41.

foreach synchronization arc $\alpha$ do

$\delta_{a i}:=\operatorname{head}(\alpha)$

add $\operatorname{les} l\left(r_{\alpha}\right) \Delta$ immediately before $\delta_{s i}$

if $\delta_{\theta i}$ has label $l$ then

remove $l$ from $\delta_{g i}$

add $l$ to test $\left(r_{\alpha}\right) \Delta$

Figure 41. Algorithm for Adding Test Instructions 


\subsubsection{Adding SET Instructions}

Adding set instructions is more difficult than adding test instructions. The reason for this is that when adding a set instruction, deadlock must be prevented. Therefore, it is essential that a set be executed for every synchronization register in the loop regardless of the path taken. If it is possible to execute an iteration of the loop without executing a set instruction, then a deadlock will occur when a test in a later iteration of the loop waits indefinitely for the set to be executed.

Each dependence in a loop is treated in turn. A set $(r)$ is first placed immediately after the source of the dependence, which is in BAS $B_{a 0}$. Each branch in the loop is then examined. If one of its targets is $B_{80}$, then we must insure that if any of the other branches is taken a set $(r)$ is also executed. Each target block, $B_{T}$ is examined. If $B_{T}$ can reach $B_{80}$ then a set cannot be placed in $B_{T}$ because the set may execute before the source executes. If a set is placed in all the other $B_{T}$ then regardless of the path taken either the set in $B_{\theta 0}$ or a set in some $B_{T}$ will execute. Figure 42 gives the algorithm.

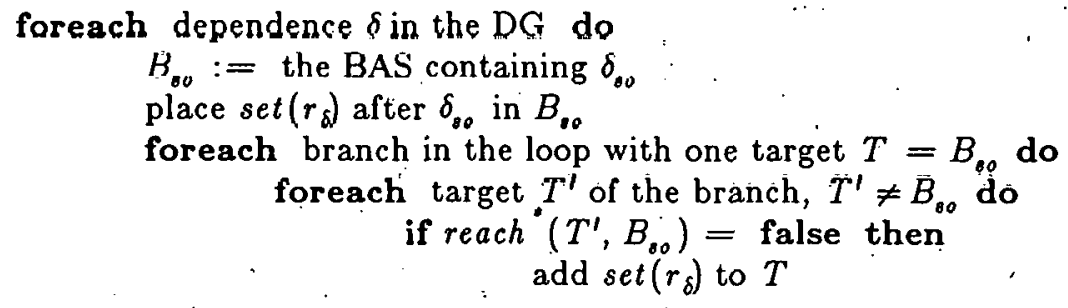

Figure 42. Algorithm for Adding set Instructions 


\section{CHAPTER 4}

\section{DEPENDENCE ARC ELIMINATION AND DEPENDENCE FOLDING}

\subsection{Introduction}

In this chapter we discuss an optimization for reducing the amount of synchronization needed in a parallel loop. Dependence arc elimination is not necessary to produce correct code. If desired it should be done immediately before the code generation algorithms of Chapter 3 . This chapter uses the modified DG constructed in Chapter 2, and produces a DG used by Chapter 3.

We first tell how to perform dependence are elimination in BAS, and using this prove several properties about it: It is then shown how to éliminate dependences within loops which have flow of control branches.

Dependence arc elimination involves determining what dependences can be safely ignored when generating synchronization instructions: Dependence arcs which can be ignored are called redundant, and are said to be covered by zero or more other dependences in the DG. A dependence arc is redundant if it is forced to be honored by either the covering dependences or a combination of the covering dependences and the control structure of the target architecture.

Dependence arc elimination is important for two reasons. The most important reason is that every instruction that is executed in a loop body uses some finite resource. Consequently, if synchronization instructions are generated needlessly for redundant dependences, resources are consumed needlessly: In particular, since some types of synchronization imply a serialization of the execution of a loop body, this needless synchronization will increase the running time of the loop. Of less practical importance is the restriction placed on the fixed number of dependences that can be synchronized by the number of synchronization registers. Since each dependence in 
the DG to be synchronized requires one synchronization register, loops with more dependences than synchronization registers cause problems. It is possible that after dependence arc elimination the number of dependences to be synchronized falls within acceptable limits.

The need to synchronize dependences arises from having to force some data accesses to follow the same order they would on a serial architecture. As some dependences are/synchronized, access to data is restricted and becomes closer to a serial order. As data accesses tend more and more towards a serial access, it becomes likely that some dependences that have not yet been synchronized will be forced to be honored by the synchronization of other data dependences along with the control structure of the architecture. These dependences are redundant and should be eliminated. It should be noted that the elimination of a redundant dependence arc does not make a loop less parallel than it would be otherwise.

Other work on this problem has been done by $\mathrm{Li}$ [LiZh85]: Our solution differs from theirs in three major ways. First, we separate the architecturally dependent portion of dependence arc elimination from the architecturally independent portion. Second, our algorithm, with the algorithms in Section 3.1, works on code segments with arbitrary flow of control graphs. Third, our algorithms allow several dependences to be used to eliminate another dependence arc.

Later in this chapter, a related subject, dependence folding, is covered. Dependence folding involves introducing new dependences to eliminate other dependence arcs. The goal of dependence folding is to reduce the number of synchronization registers needed. Dependence folding can cause loops to be more serial than necessary.

\subsection{Definitions and Basic Strategies}

A dependence arc with distance $\Delta$ gives a constraint on the legal execution 'of a program segment: $\Delta$ iterations of the loop must execute between the source and sink of the dependence. When the dependence has been synchronized, there now exists a controlled path from one statement to another across $\Delta$ iterations. A controlled path arc, labeled with distance $\Delta$ from $S_{i}$ to 
$S_{j}$ asserts that all dependences from $S_{i}$ to $S_{j}$ of distance $\Delta$ will be enforced. Thus, while a dependence represents a condition that must be satișied, a controlled path represents a condition that is satisfied, thereby forcing the dependence to be honored.

When a loop is synchronized two things happen. The first is that synchronization statements are placed in the loop. The second is that dependences in the loop are replaced by controlled paths from synchronization statement to synchronization statement in the loop. The loop can now be represented by a Controlled Path Graph, or CPG. Nodes in the CPG will either be synchronization nodes, which represent synchronization statements, or source nodes, which represent the original program source statements. Arcs, or controlled path arcs in the CPG will either be synchronization arcs, which represent controlled paths introduced by synchronization statements; or machine ares, which represent controlled paths introduced by the architecture of the target machine. In examples, where CPGs are depicted pictorally, synchronization arcs in the CPG will be shown as solid lines and machine arcs will be shown as dashed lines.

In the algorithms presented in this section, a CPG will be represented as a connectivity matrix. For singly nested parallel loops, a connectivity matrix $C$ will be a two dimensional matrix indexed by ordered pairs of the form $\left\langle i, S^{\prime}\right\rangle$. $C\left[\left\langle i, S^{\prime}\right\rangle,\left\langle i^{\prime}, S^{\prime}\right\rangle\right]$ has the value true if there is either a machine or synchronization arc from $S$ in iteration $i$ to $S^{\prime}$ in iteration $i^{\prime}$. The transitive closure of $C$ is $\dot{C}^{*}$. $C^{*}\left[\langle i, S\rangle,\left\langle i^{\prime}, S^{\prime}\right\rangle\right]$ is true if there exists a controlled path from statement $S$ in iteration $i$ to statement $S^{\prime}$ in iteration $i^{\prime}$. As will be seen, $C$ needs to only be large enough to contain the first $\Delta_{\max }^{L}+1$ iterations of the loop.

As before, $\Delta_{\max }^{L}$ stands for the maximum dependence distance in the loop being synchron- . ized. 


\subsection{Building a CPG in a BAS}

Building a CPG is straightforward, and the algorithm for doing so is given in Figure 43.

The process can be broken down into three distinct phases. Initially a synchronization dependence graph, or $S D G$, is built for the $D G$ by inserting nodes for the synchronization statements that must be added to the loop to synchronize it according to the basic rules of Section 3.1. Dependence arcs are removed from the SDG and replaced with controlled path arcs corresponding to the synchronization ares llial will be added to tho final CPG. Figure 44n shows a lonp,

$S_{f}, S_{l}=$ nodes corresponding to the first and last nodes in the loop

$/$ Build an SDG */.

replicate the DG as the SDG

foreach statement $S_{80}$ in the SDG do

$$
\text { foreach dependence } \delta=\left(S_{80}, S_{\theta i}\right) \text { do }
$$

$\Delta_{\delta}$ is the distance of $\delta$

place synchronization instructions on the SDG using the appropriate algorithms of Chapter 3

place an arc from each $\operatorname{set}\left(r_{\delta}\right)$ or

testset $\left(r_{\delta}\right)$ to the corresponding

$\operatorname{tes} l\left(r_{\delta}\right) \Delta_{\delta}$ labcling

the $\operatorname{arc} \Delta_{\delta}$.

$/^{*}$ bulld the conneclivily graph* *

$0:=$ fälse

$/ *$ add synchronization arcs */

access each node $n$ of the SDG in lexical order

foreach arc $\alpha$ out of $n$ do

$$
\begin{aligned}
& \Delta_{\alpha}:=\text { label of } \alpha \\
& h:=\text { head of } \alpha \\
& \text { for } i:=1 \text { to } 1+\Delta_{\max }^{L}-\Delta_{\alpha}+1 \text { do } \\
& \qquad C\left[<i, n>,<i+\Delta_{\alpha}, h>\right]:=\text { true }
\end{aligned}
$$

${ }^{*}$ add machinc arce *

$$
\begin{aligned}
& \text { for } i:=1 \text { to } \Delta_{\max }^{L}+1 \text { do } \\
& \quad \text { for } n:=S \text { to } S_{l}-1 \text { do } \\
& \qquad C[<i, n>,<i, n+1\rangle]:=\text { true }
\end{aligned}
$$

Figure 43. Alyorithm for Building a CPG 
Figure 44b shows the DG for the loop, and Figure 44c shows the SDG for the loop. The first part of the algorithm in Figure 43 gives the details of how this is done. The primary purpose of building the SDG is to be able to determine the location of potential synchronization instructions in the final loop, and to be able to see the positions of controlled path arcs relative to the sources and sinks of all dependences in the loop.

If it is only desired to determine how many dependences are inherently redundant, then the step of the algorithm where a SDG is built for the DG can be skipped. This means that dependence arcs are to be treated as controlled path arcs, and the DG is to be used as the SDG. This would also be a valid SDG for a synchronization instruction set in which the store or fetch of a variable would be done by the synchronization instruction itself. In any case; the dependence arc to be removed should not be placed in the SDG.

10

$$
\begin{array}{cc}
\text { DOSPREAD } 10 \mathrm{I}=1, \mathrm{~N} & \\
\mathrm{~A}(\mathrm{I})=\mathrm{B}(\mathrm{I})+\mathrm{C}(\mathrm{I}) & S_{1} \\
\mathrm{C}(\mathrm{I})=\mathrm{A}(\mathrm{I}-1)+\mathrm{D}(\mathrm{I}) & S_{2} \\
\mathrm{D}(\mathrm{I}+1)=\mathrm{E}(\mathrm{I})+\mathrm{C}(\mathrm{I}-2) & S_{3} \\
\text { CONTINUE } &
\end{array}
$$

(a) A Loop

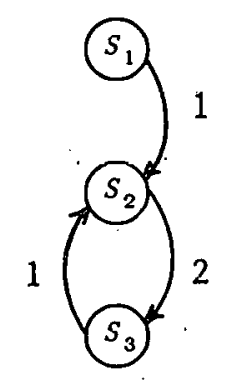

(b) The DG for (a)

Figure 44. A SDG Example 


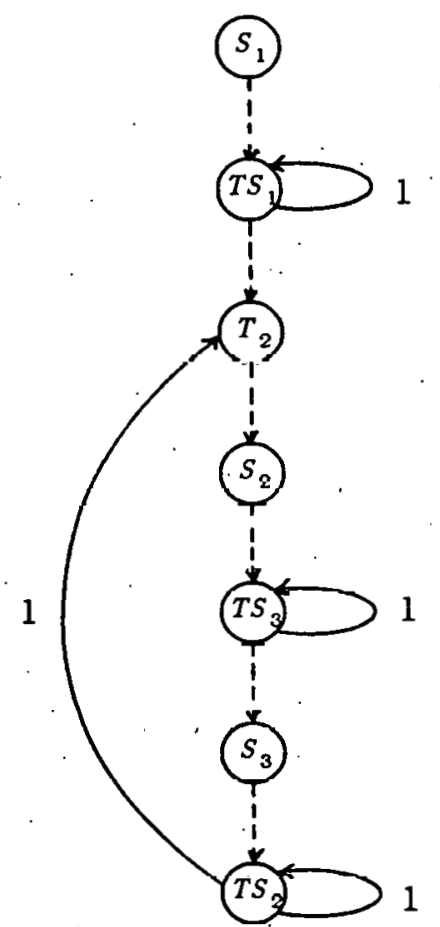

(c) The SDG for (a)

DOSPRE $\Lambda \mathrm{D} 10 \mathrm{I}-1, \mathrm{~N}$

$A(I)=B(I)+C(I)$

TESTSET(1)

$\operatorname{TEST}(2) 1$

$\mathrm{C}(\mathrm{I})=\mathrm{A}(\mathrm{I}-1)+\mathrm{D}(\mathrm{I})$

TESTSET(3)

$\mathrm{D}(\mathrm{I}+1)=\mathrm{E}(\mathrm{I})+\mathrm{C}(\mathrm{I}-2)$

10 CONTINIJF

TESTSET(2)

$S_{1}$

$T S_{1}$

$T_{2}$

$S_{2}$

$T S_{3}$

$S_{3}$

$T S_{2}$

(d) Loop Corresponding to the SDG of (c)

Figure 44. Continued

Once the SDG has been built it is simple to construct the CPG. First we observe that only iterations 1 to $\Delta_{\text {max }}^{L}$ need be included in the CPG. This will be shown later, after we prove the condition necessary to allow a controlled path arc to be eliminated. 
Each node in the SDG is examined in turn. Each arc out of a node represents a controlled path, since all dependences were replaced with the synchronization necessary to enforce the dependence. Thus, for each arc in the SDG from $S$ to $S^{\prime}$ of length $\Delta$, we add an arc from every $\langle i, S\rangle$ to every $\left\langle i+\Delta, S^{\prime}\right\rangle$. This arc is labeled. with the number of iterations over which it extends. With arcs going from a set to a test, or from a testset to a test, this distance is the dependence distance, and corresponds to the value of $\Delta$ which will be appended to the test instruction. When going from a testset to itself, the value is 1 , corresponding to $\Delta=1$ in the implied test instruction. This takes care of all of the synchronization arcs. Figure 45a shows the partially constructed CPG for the SDG of Figure 44c with synchronization arcs added.

The machine arcs are' more straightforward. Since the loop is a BAS, $S_{i+1}$ always executes after statement $S_{i}$. Since stores and fetches execute serially on a single processor, data from within an iteration for $S_{i+1}$ must be ready when $S_{i}$ has completed. Therefore a machine arc can be placed from $S_{i}$ to $S_{i+1}$. Figure $45 \mathrm{~b}$ shows the completed CPG.

If our architecture was different, or loop iterations were executed differently, we would have to place machine arcs differently. For example, if the execution of a single iteration was spread across different processors [Veid85] then it would no longer be true that stores and fetches within an iteration occurred in a serial fashion. Stores and fetches for that part of an iteration executing on a single processor would occur in a serial order, but no assumptions could be made about order of stores and fetches in relation to stores and fetches on different processors.

An even more extreme example is given by Zhu and Yew in [ZhYe84]. In their model, store and fetch requests occur. in order, but the order in which stores and fetches actually occur depends upon the conflicts encountered in the interconnection network. No assumptions can be made about fetches and stores within a single processor, and therefore no machine arcs can be placed in the CPG. 


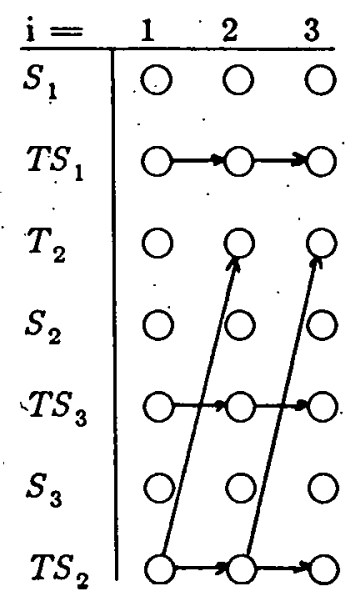

(a) Partially Constructed CPG for the DG of Figure 44

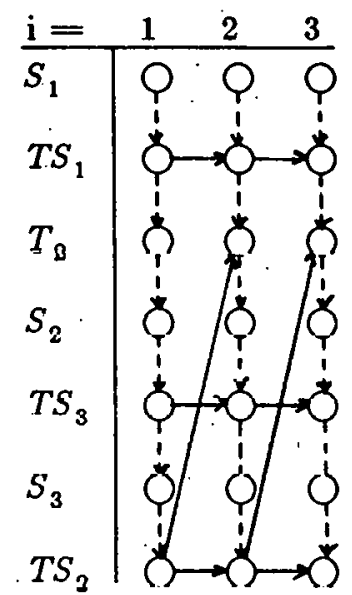

(b) The Completed DG

Figure 45 A ripr.

The difficult part of determining the placement of synchronization and machine arcs for a new architecture is mapping the semantics of the instructions and control structure onto control arcs in the graph. Once this is done, building the CPG automatically is fairly easy. The conservative approach is to leave out ares when in doubt. This underestimates the constraints placed on the program execution by the architecture and synchronization, and potentially leads to unnecessary synchronization being added. This leads to slower running times, but the running 
times are no slower than if dependence arc elimination had not been tried, and the execution of the program is correct. In any case, this sort of analysis is necessary to determine what dependences need synchronizing and how they might be legally synchronized.

\subsection{Dependence Arc Elimination in a BAS}

To eliminate some synchronization arc $\alpha$, and the synchronization instructions that form it, it is only necessary to show that there exist at least one path in the CPG from the source to the sink of $\alpha$, that does not include $\alpha$. In other words, a controlled path arc can be eliminated if a controlled path not containing it performs the same function. To see if this condition holds requires only that the we find an alternate path from the source to the sink of the dependence in the CPG. This can be done very easily by using the transitive closure of the connectivity matrix $\mathrm{CPG}, \mathrm{C}^{*}$

Having computed $C^{*}$, determining whether an alternate path exists from the source to the sink of the dependence arc being eliminated is straightforward. If $S_{s o}$ is the source of a dependence in the controlled path arc to be eliminated, $S_{\theta i}$ is the sink of the same dependence, and $\Delta_{\alpha}$ is the distance of the dependence, then an alternate path exists if $C^{*}\left[<1, \delta_{s o}>,<\Delta_{\alpha}+1, \delta_{e i}>\right]=$ true

We now prove that finding an alternate controlled path is necessary and sufficient for eliminating a dependence arc.

Lemma 1: The CPG is acyclic.

Proof: All arcs in the SDG are either LBDs or LFDs. All dependence distances attached to an arc will either be $<0,=0$, or $>0$. From this, we get the following four cases:

case 1: An LBD with $\Delta \leq 0$. Arcs of this type cannot be added to the graph as this would require the flow of data from a statement to a lexically prior 
statement in either the same or an earlier iteration. Note that if backwards branches were allowed, this would not be true.

case 2: An LFD with $\Delta<0$. Arcs of this type cannot be added to the graph, as it would require the flow of data from a statement to a lexically forward statement in an earlier iteration.

case 3: An LBD with $\Delta>0$. Such an arc can only form a cycle if we have at least one arc of the type in case 1 or case 2 . Since such arcs cannot, appear in the CPG, we cannot have a cycle from arcs of this type.

case 4: An LFD with $\Delta \geq 0$. Such an arc can only form a cycle if we have at least one arc of the type in case 1 . Since such arcs cannot be in the CPG, arcs of this type cannot cause a cycle.

Since no other arcs can exist, the CPG must be acyclic.

Lemma 2: All CPG of length $\Delta$ constructed using the Algorithm of Figure 43 for some SDG will be isomorphie with respect to onc nnother.

Proof: Assume two graphs have been constructed using the Algorithm. All arcs into and out of a node depend on three things: the structure of the SDG, the distance of the node from the first iteration in the graph and the statement the node represents. Clearly, if a node is $\gamma$ iterations from the first node in the graph in both graphs, then the nodes coming into and out of the node will be identical. If the first iteration of the first graph is $\iota$, and the lirsi ileralion of the secund graph is $\iota$, lhen , we can shift all iterations in both graphs by $\imath$. Therefore the node in both new graphs will be at $\gamma$, and will be indistinguishable. A similar argument can be constructed for each node.

Theorem 1: A controlled path arc $\alpha$ can be removed from an CPG if and only if for each dependence $\delta$ covered by $\alpha$ there exists a controlled path arc from the source of $\delta$ in 
iteration 1 of the CPG to the sink of $\delta$ in iteration $1+\Delta_{\delta}$

Proof: We first show that the condition is sufficient. Let $\delta_{\theta 0}$ be the source of some dependence covered by $\alpha$, and $\delta_{\varepsilon i}$ be the sink of some dependence covered by $\alpha$. Now. assume that there is a path over $\Delta_{\delta}$ iterations from $\delta_{s o}$ to $\delta_{a i}$. This path consist of . arcs, in the order traversed, $A_{1}, A_{2}, \cdots, A_{n}$, none of which is $\alpha$. By definition, no $A_{i}$ can be traversed until $A_{i-1}$ has been traversed. But $A_{i}$ cannot be traversed until $\delta_{\theta 0}$ has executed, thus $A_{n}$ cannot execute until $\delta_{\theta 0}$ has executed. Since $\delta_{\theta i}$ cannot execute until after $A_{n}$ has been traversed, $\delta_{\theta i}$ cannot execute until after $\delta_{80}$. Therefore the dependence is satisfied. By Lemma 1, the graph is acyclic. Therefore we need not worry about any iterations less than iteration 1 or greater than $\Delta_{\alpha}+1$. By Lemma 2, the iterations can be renamed, thus the dependence is satisfied for all iterations.

The condition must be necessary, for if there is no controlled path from the source to the sink of the dependence, then nothing exists to force the sink to execute after the sourcc.

\subsubsection{Dependence Arc Elimination is NP-Hard}

We now show that dependence arc elimination in a SDG is NP-Hard. The proof proceeds by first showing that elimination of a dependence arc in an acyclic DG is NP-hard. This result is then used to show that dependence arc elimination in an acyclic SDG is also NP-hard. Both of these are shown by mapping the elimination of a single dependence arc onto the Partition problem . [CaJo79|.

The Partition problem can be stated as follows: Let $A=\left\{\alpha_{1}, \alpha_{2}, \cdots, \alpha_{n}\right\}$, where each $\alpha_{i}$ is an integer. The problem is solved if the question:

Do there exist two subsets of $A, A^{\prime}$ and $A-A^{\prime}$, such that $\sum_{\alpha \in A^{\prime}} \alpha=\sum_{\alpha \in A-A^{\prime}} \alpha$ 
is answered. Figure 46 gives an example of some $A$ for which the answer would be "yes".

We map this problem onto dependence arc elimination in the following manner: Let $A$ be as above. The SDG of Figure 47 is built for $A$. The lengths of the dependence labeled $\alpha_{i}$ is $\alpha_{i}$. The arc to be eliminated is the one of length $M / 2$, where $M=\sum_{\alpha \in A} \alpha$. Next a CPG and $\mathrm{CPG}^{*}$ are built for the SDG. If a path exists from $\langle 1, N\rangle$ to $\left\langle M / 2, N_{n+1}\right\rangle$ then there exists a valid partition of $A$. $A^{\prime}$ contains those non-zero arcs used in getting from $N$ to $N_{n+1}$, and $A-A^{\prime}$ contains those non-zero arcs not used. Since the total number of iterations that can be covered is $M$, and we covered $M / 2$ of them, the arcs not traversed must also cover $M / 2$. Two things should be noted. The first is that taking a zero arc is the same as leaving the corresponding $\alpha$ out of $A^{\prime}$. The second is that each arc can only be used once, as required in the Partition problem. This is because the absence of any LBDs in the SDG prevents a path existing from an occurrence of some $\alpha_{i}$ in some iteration to another occurrence of $\alpha_{i}$ in a later iteration. Therefore $\alpha_{i}$ can only be used once.

We now consider the case of cyclic SDGs. Since we never know in advance if a back arc is useful in eliminating a dependence arc, we can make the length of the back arc any value. Figure 47 is modified by adding a back arc of length $(M / 2)+1$. Since we only wish to traverse

$$
\begin{aligned}
& A=\{2,3,5,6\} \\
& M=16
\end{aligned}
$$

(a) The Set $A$ and $M$ for $A$

$$
\begin{aligned}
& A^{\prime}=\{2, b\} \\
& A-A^{\prime}=\{3,5\}
\end{aligned}
$$

(b) A Partition of $A$

Figure 46. An Example of a Partitionable Set 


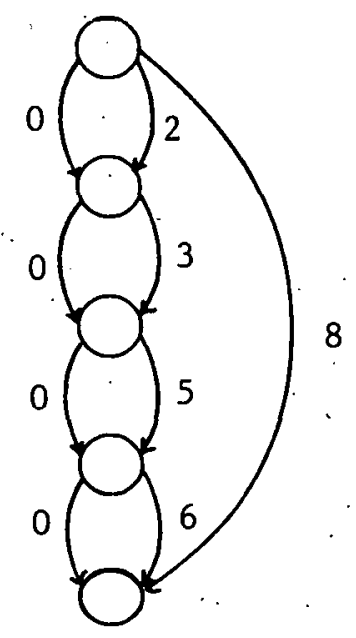

Figure 47. A SDG Equivalent $A$ of Figure 46

$M / 2$ iterations, the back arc will never be used. Therefore eliminating the dependence arc $M / 2$ is the same as before. Thus dependence arc elimination is NP-Hard in general for both cyclic and acyclic SDGs.

\subsection{Dependence Arc Elimination with Flow of Control}

When the loop body over which dependence arc elimination takes place contains flow of control branches, we must account for this flow of control in eliminating dependence arcs. The problem that arises with flow of control branches is that the controlled paths that can possibly be taken during an iteration of the dospread loop can depend on what statements are executed in that path. Consider, for example, the program of Figure 48a. Figure 48b gives the CPG for the loop assuming that the IF statement always takes the true path. Since statements $S_{3}$ through $S_{8}$ are never taken, there cannot exist controlled paths between those statements. Figure $48 \mathrm{c}$ gives the CPG for the loop assuming that the IF statement always takes the false branch. In this case statements $S_{9}$ through $S_{13}$ are 
never taken. In both cases, however, we can eliminate the dependence.

In Figure 48d we see a CPG for the loop when the true path is taken for the first four iterations, and then the false path is taken for the rest. Note in this case we cannot eliminate the dependence, because from iteration four we cannot get to iteration seven using controlled paths of length two. Thus we see that the existence of a single controlled path through the loop is not sufficient, nor is the existence of controlled path through every flow of control path in the loop. Instead, what must be shown is that there is a controlled path through the loop for every combination of flow of control paths possible when executing the loop for iterations one through $\Delta_{\max }^{L}$. Figure 47 gives an algorithm for determining all the possible flow of control paths through a loop.

10

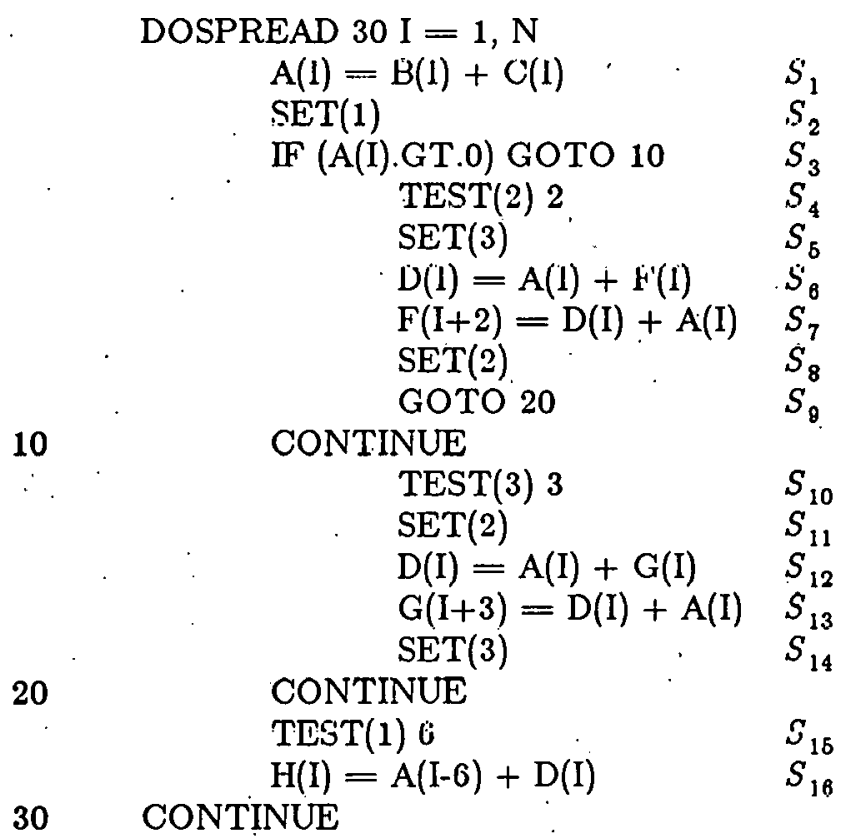

30

(a) A Synchronized Loop

Figure 48. Problems Arising from Dependence Arc Elimination with Branches. 
73

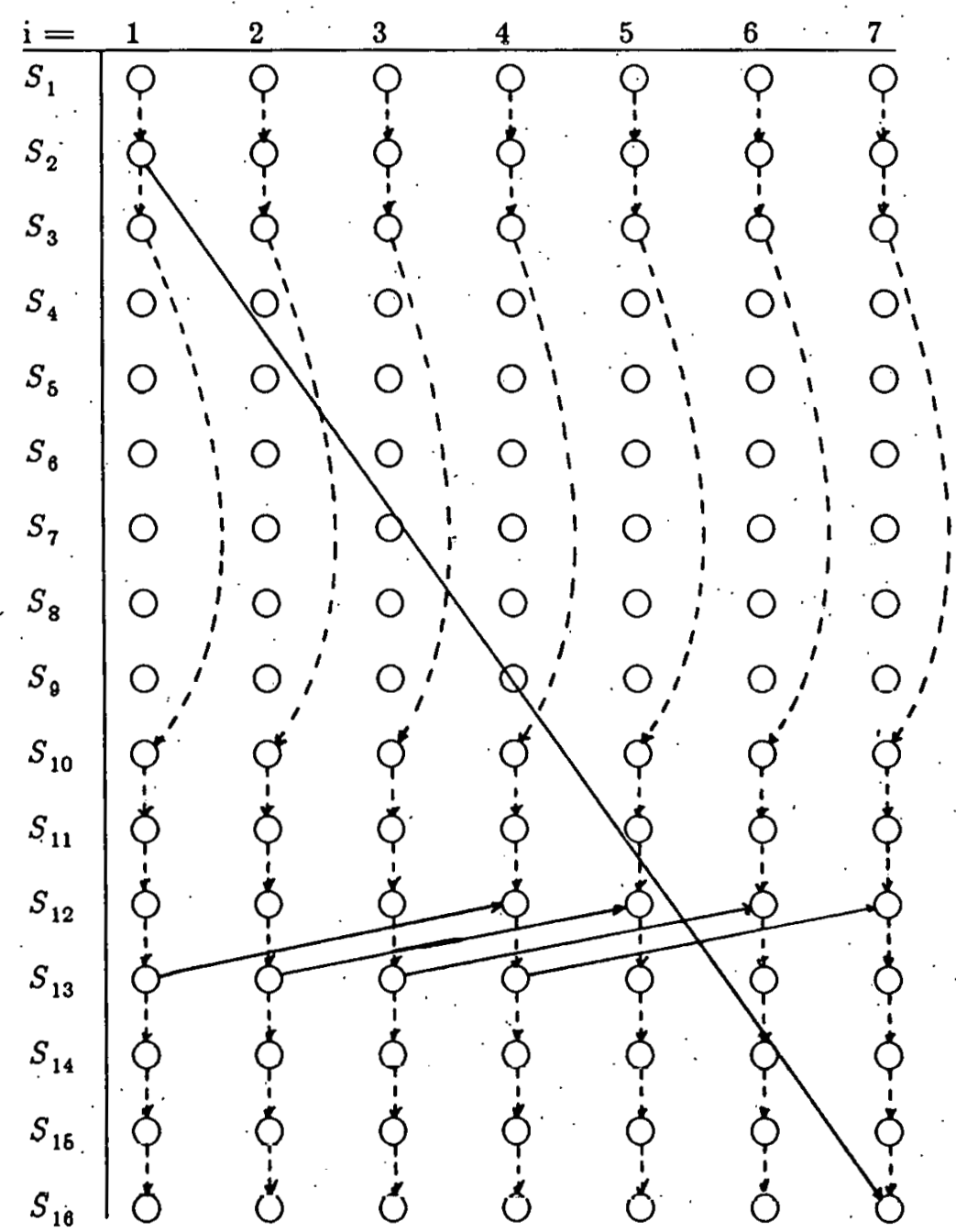

(b) CPG for (a) when only the True Path is taken.

Figure 48. Continued 


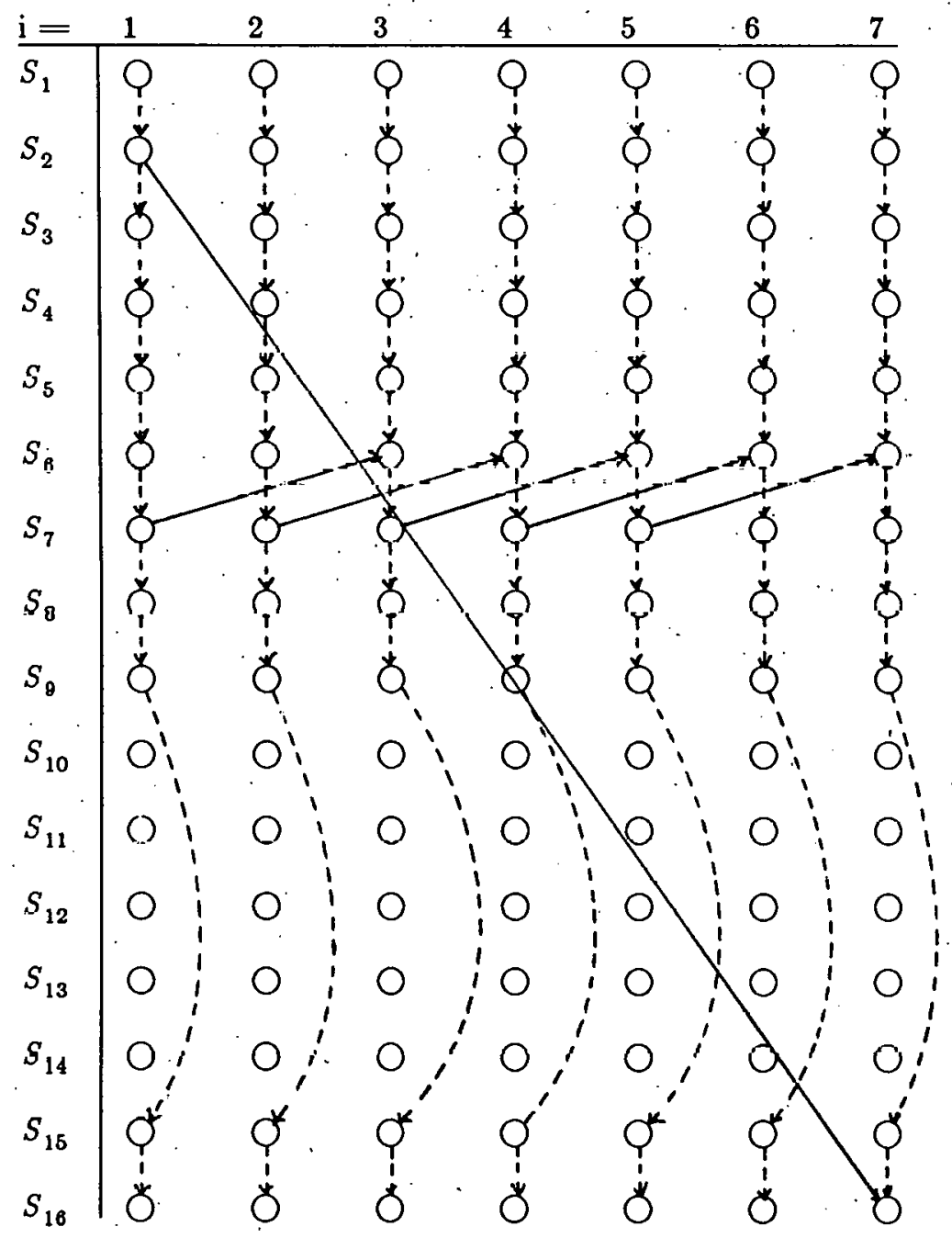

(c) CPG for (a) when only the False Path is Taken

Figure 48. Continued 

1 max paths - maximum number of flow of control paths through a loop max」ength - maximum number of statements in any flow of control path num_stmts - number of statements in the loop.

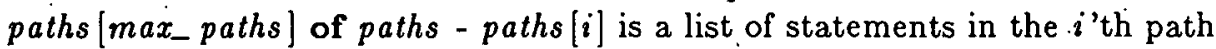
num $m_{-}$paths - number of How of control paths through the loop body found so far this_path [max_length] of statements - path currently being found

find_all_paths (this_path, length, $S$ )

length - length of path currently being found

leaf - true if no statements are executed after $S$ in the loop

$S$ - current statement in reach,

length := length +1

this_path [length]:=S

leaf := true.

for $i:=1$ to $n$ um_stmts do

if $\operatorname{reach}_{\mathrm{s}}(S, i)$ then

leaf $:=$ false

if leaf then

find_all_paths (this__path, length, i)

end find_all_paths

num_paths := num_paths +1

paths $[$ num_paths $]:=$ this_ path

num_palhs $:=0$

find_all_ paths (this_path, $0, S_{1}$ )

Figure 47. Algorithm for Finding all Flow of Control Paths through a Loop Body

When all the possible flow of control paths through the loop have been found, a CPG is built for each combination of flow of control paths over the iteration range being considered for dependence arc elimination. If $P$ is the number of flow of control paths being constructed, then $\Delta_{\max }^{L\left(l^{\prime}\right)}$. CPGs must be generated and checked. Fortunately, most dependence distances are two or less, so this is not as bad as as it might initially seem. Figure 49 gives an algorithm for building a CPG using flow of control information. Note that when building the CPG when flow of control is being considered that only nodes which are executed in a particular iteration are at the head or tail of a controlled path. 
$\delta_{e}$ is the dependence arc being eliminated

$S D G$, palhs, path」list are assumed already built

$C:=$ false

$/$ add synchronization arcs * /

access each node $n$ of the SDG in lexical order

for each $\operatorname{arc} \alpha$. out of $n$ do

$\Delta_{\alpha}:=$ label of $\alpha$

$h:=$ head of $\alpha$

for $i:=k$ to $k+\Delta_{\max }^{L}-\Delta_{\alpha}+1$ do

$/^{*} \mathrm{i}$ is the iteration of the tail of the synchronization $\operatorname{arc}^{*} /$

$/^{*} \mathrm{i}$ sub $\mathrm{h}$ is the iteration of the head of the synchronization arc $* /$

$i_{h}:=i+\Delta_{\alpha}$

if $n$ in paths $[$ path_ist $[i]]$ and $i_{h}$ in paths $\left[\right.$ path_list $\left[i_{h} \mid\right]$ then

$C\left[\langle i, n\rangle,\left\langle i_{h}, h\right\rangle\right]:=$ true

$l^{*}$ add machine arcs *

for $i:=k$ to $k+\Delta \Delta_{\max }^{L}+1$ do

for $n:=N_{f}$ to $N_{l}$ do

for $t:=n+1$ to $N_{l}$ do

if $\operatorname{stmt}$ reach $(n, t)$ and $n$ in paths [path_list $[i]]$

and $t$ in paths [path_list $[i]]$ then

$C[\langle i, n\rangle,\langle i, t\rangle]:=$ true

Figure 49. Algorithm for Building a CPG with Flow of Control Information

If the dependence arc is redundant over all possible combinations of flow of control paths, then the arc maylbe eliminated. The algorithm for dependence arc elimination taking flow of control into account is given in Figure 50. The degenerate case of this algorithm, where no branches exist in the loop body, is the same as the algorithm for dependence arc elimination in a BAS.

\subsection{Optimal Dependence Arc Elimination}

Consider the loop of Figure 51a, and its associated CPG in Figure 51b. Neither of the dependences in the loop can be eliminated. If the code is rearranged, as in Figure 51c, yielding the CPG of Figure 51d, then one dependence can be eliminated. Therefore the algorithm we 
max_paths - maximum number of paths through the loop

paths [max paths] - paths $[i]$ contains a list of all statements in the $i$ 'th path path」ist $\left[\Delta_{m a x}^{L}-\right.$ path_list $[i]$ is the path taken on the $i$ 'th iteration

num $m_{-}$paths - number of entries in paths

redundant - true if dependence is redundant, false otherwise

reach $_{\sigma}$ is a statement level reachability matrix

$\delta$ - the dependence arc being checked for redundancy.

$\delta_{v e}, \delta_{z i}$ - the source and sink of $\delta$

this_path $\left[\Delta_{\text {max }}^{L}\right]$ - dummy parameter for find_all_paths

check_dep (pati_list, iteration)

$C$ is a $C P G$, and $C$ is its transitive closure

if iteration $>\Delta_{\max }^{L}$ then

build a $\mathrm{C}$ using the algorithm of Figure 49

$C:=$ transitive_closure $(C)$

if not $C\left|\delta_{s o}, \delta_{s i}\right|$ then

redundant $:=$ false ,

else

for $i:=1$ to $\Delta_{\max }^{L}$ do path」ist [iteration] : $=i$

end check_dep check_dep (path_list, iteration + 1)

find all flow of control paths using the algorithm of Figure 47

build an SDG by duplicating the DG and adding synchronization nodes

foreach synchronization arc $\delta$ in the SDG do

redundant $:=$ true

check_dep (path」ist, 0$)$

if redundant $=$ true then

remove $\delta$ and its associated synchronization nodes from the SDG

Figure 50. Dependence Arc Elimination with Flow of Control

present is optimal in the number of dependences only for a given code arrangement, and is not optimal for all semantically correct executions of the loop. The rules for determining the optimal number of dependence arcs given the possibility of code motion are beyond the scope of this paper. 
10

$$
\begin{array}{rr}
\text { DOSPREAD } 10 \mathrm{I}=1, \mathrm{~N} & \\
\mathrm{~A}(\mathrm{I})=\mathrm{B}(\mathrm{I})+\mathrm{C}(\mathrm{I}) & S_{1} \\
\mathrm{~B}(\mathrm{I})=\mathrm{A}(\mathrm{I}-1)+\mathrm{E}(\mathrm{I}) & S_{2} \\
\mathrm{~F}(\mathrm{I})=\mathrm{G}(\mathrm{I})+\mathrm{H}(\mathrm{I}) & S_{3} \\
\mathrm{P}(\mathrm{I})=\mathrm{F}(\mathrm{I}-1)+\mathrm{Q}(\mathrm{I}) & S_{4} \\
\text { CONTINUE } &
\end{array}
$$

(a) A Loop

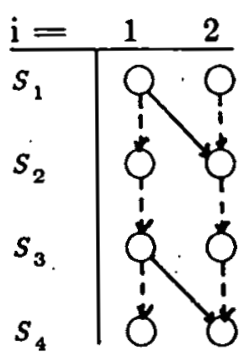

(b) The CPG for (a)

DOSPREAD $10 \mathrm{I}=1, \mathrm{~N}$

$$
\begin{array}{ll}
\mathrm{A}(\mathrm{I})=\mathrm{B}(\mathrm{I})+\mathrm{C}(\mathrm{I}) & s_{1} \\
\mathrm{~F}(\mathrm{I})=\mathrm{G}(\mathrm{I})+\mathrm{H}(\mathrm{I}) & s_{3} \\
\mathrm{P}(\mathrm{I} 0=\mathrm{F}(\mathrm{I}-1)+\mathrm{Q}(\mathrm{I}) & s_{4}
\end{array}
$$

10 CONTINUE

(c) The Loop of (a) with the Statements Rearranged

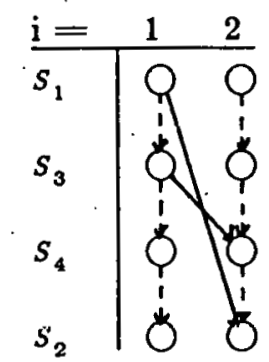

(d) The CPG for (c)

Figure 51: Optimal Dependence Arc Elimination Requiring Code Motion 


\subsection{Dependence Arc Elimination in Nested Parallel Loops}

The same techniques used to eliminate dependences with only one level of parallel loops can be used to eliminate dependences with multiple levels of nested loops. It is beyond the scope of this thesis to give details on the placement of synchronization in multiply nested parallel loops. Therefore, we will discuss dependence arc elimination from a SDG which is identical to the DG for the loop.

With unnested parallel loops, CPG is a function $f:\left[\langle I, S\rangle,\left\langle I^{\prime}, S^{\prime}\right\rangle\right] \rightarrow$ boolean. With multiply nested parallel loops, the CPG has as its domain $\left(<I_{1}, I_{2}, I_{3}, \cdots I_{n}, S>, I_{1}{ }^{\prime}, I_{2}{ }^{\prime}, I_{3}{ }^{\prime}, \cdots I_{n}{ }^{\prime}, S^{\prime}>\right.$ ), where $n$ is the level of nesting of the parallel loop. In a proof similar to that given for Theorem 1 it can be shown that the CPG must be acyclic; this is a result of dependence distances on the outermost parallel loop having to be $\geq 0$. It is also true that the CPG need only have iterations from 1 to $\Delta_{m a x}^{L}$ in it for each parallel loop $L$ in the loop nest.

Figure 52a gives a loop nest with two parallel loops. Figure 52b gives the DG for the loop. The first number on each dependence arc is the dependence distance on the outer loop and the second number is the dependence distance on the inner loop. Figure 52c gives a pictoral version of the CPG for the loop.

$\Lambda$ gain, eliminating a dependence requires finding an alternate controlled palls frum the source to the sink of the dependence. By following the dependence with a distance of $\langle 1,-1\rangle$ from $I$ iteration to $I$ iteration, and the zero length machine arcs from statement to statement within a $J$ loop, we can go from the source to the sink of the dependence with a distance of $<2,-2\rangle$ without traveling on this arc. Therefore it can be eliminated.

\subsection{Dependence Folding}

It may be that after dependence arc elimination there are still too many dependences to be synchronized with the available number of synchronization registers. If so, it is possible to 


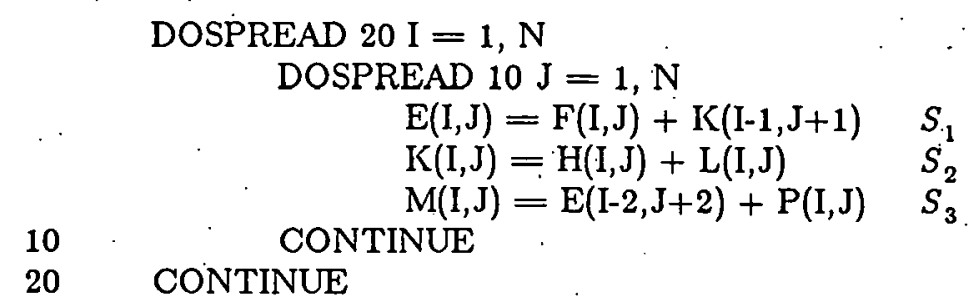

(a) A Loop Nest

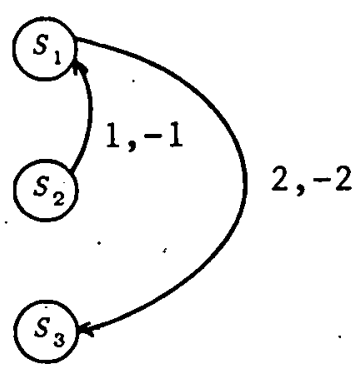

(b) DG for (a)

Figure 52. Dependence Arc Elimination in Nested Parallel Loops

eliminate other dependences by adding dependences. This process is called dependence folding, and the dependences being eliminated are said to be folded into the added dependence.

In dependence folding, we replace two or more dependences with one new onc. Rules for dependence folding vary from architecture to architecture, and are formulated by considering what types of dependences can be added that will cause the old dependences to be eliminated. We present here some ideas on dependence folding for the two synchronization instruction sets of this thesis.

Since LBDs cannot by eliminated by LFDs with the instructions and architectures discussed in this thesis, folding LBDs will require the addition of extra LBDs. LFDs can only be eliminated by a new LFD under one circumstance. 


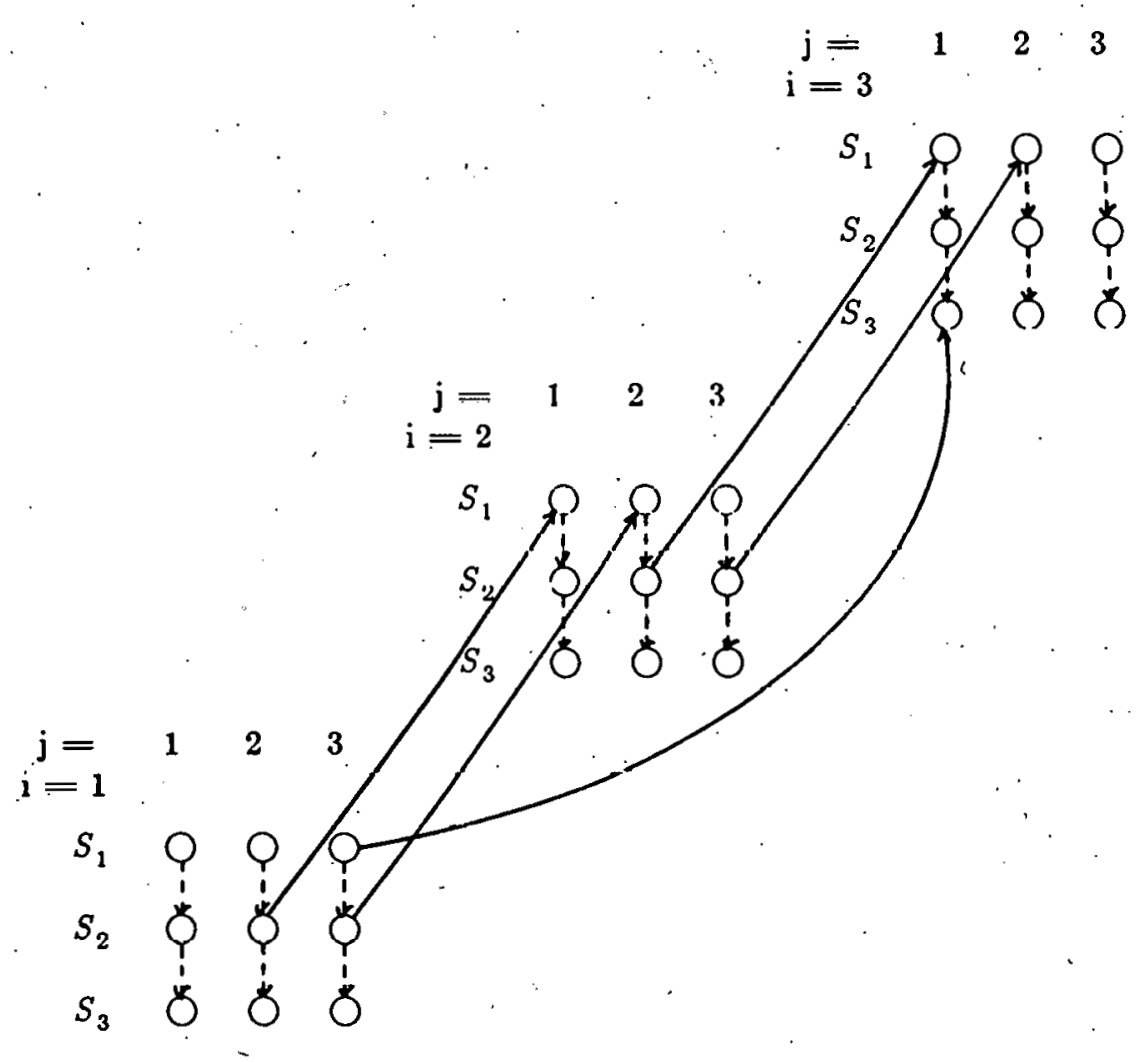

(c) CPG for (b)

Figure 52. Cunlinued

If all the LFDs have the same distance (or testset instructions are used), and if the dependences overlap, then folding is very easy. Two LFDs overlap if the source of the lexically last precedes the sink of the lexically first LFD. Let $\delta_{1}$ be the first dependence, $\delta_{2}$ be the second, and $s o_{1}, s i_{1}, s o_{2}, s i_{2}$ be their sources and sinks. Also lel $s 0_{1} \leq s 0_{2}<s i_{1} \leq s i_{2}$. A new dependence is added, of length $\Delta_{\delta_{1}}$ from $s o_{2}$ to $s i_{1}$, and $\delta_{1}$ and $\delta_{2}$ are removed from the DG. Figure 53a shows two LFDs with equal $\triangle \mathrm{s}$ folded into a single LFD.

If this condition cannot be met, then the LFDs must be eliminated with an LBD. 
10

$\begin{aligned} \text { DOSPREAD } 10 \mathrm{I}=1, \mathrm{~N} & \\ \mathrm{~A}(\mathrm{I})=\mathrm{B}(\mathrm{I})+\mathrm{C}(\mathrm{I}) & S_{1} \\ \mathrm{~B}(\mathrm{I})=\mathrm{C}(\mathrm{I})+\mathrm{D}(\mathrm{I}) & S_{2} \\ \mathrm{E}(\mathrm{I})=\mathrm{A}(\mathrm{I}-2)+\mathrm{C}(\mathrm{I}) & S_{3} \\ \mathrm{~F}(\mathrm{I})=\mathrm{B}(\mathrm{I}-2)+\mathrm{C}(\mathrm{I}) & S_{4}\end{aligned}$

(a) A Loop

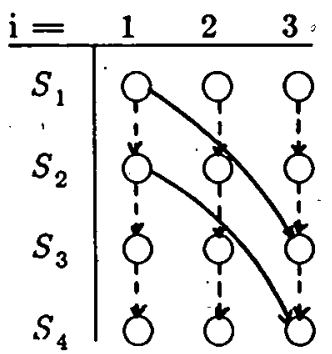

(b) The CPG for (a)

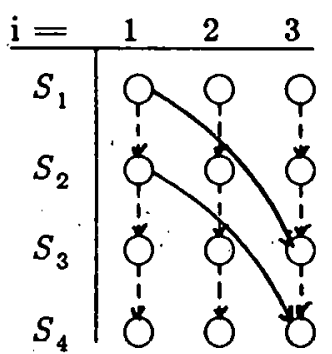

(c) The CPG of (b) after Folding

Figure 53. Folding Two LFDs with Equal $\Delta s$ into a single LFD

To fold $n$ LBDs, or LFDs that do not meet the above conditions, into a single LBD, we place an LBD from the last source or sink to the first source or sink. The distance, $\Delta_{l b d}$, attached to the LBD should be the greatest common divisor, or GCD, of all the dependences to be folded. The distance is the GCD since there exists some integer $C_{i}$ for dependence $i$ being eliminated, such that $C_{i} \cdot \Delta_{l b d}$ equals the distance of dependence $i$. Figure 54 shows two LBDs folded into a single LBD. Figure 55 shows two LFDs folded into a single LBD. 


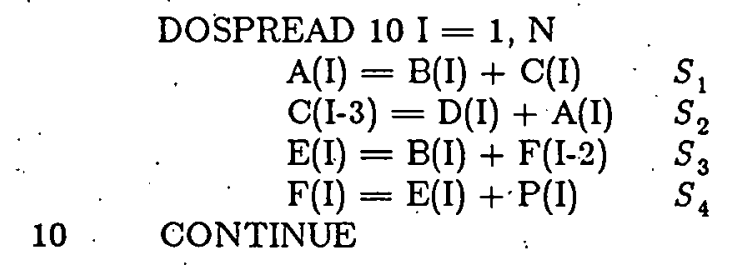

(a) A Loop

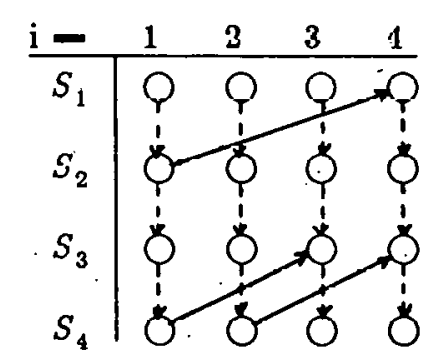

(b) The CPG for (a)

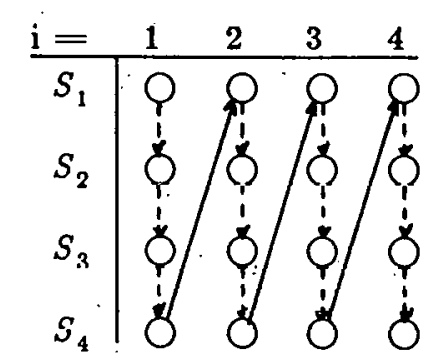

(c) The CPG of (b) after Folding

Figure 54. Folding Two LBDs into a single LBD

In both cases, the dependences being folded can be removed from the graph.

In practice adjacent pairs of dependences should be folded, since this minimizes the lcxical length of the serial portion of the loop. The loop should be timed for all such pairings being. folded. Then the pair that gives the lowest time should be picked for folding. As with all compile-time timings, the results will only be approximate. This should be repeated until the number of dependences is equal to the available number of synchronization registers. As a general rule, as many registers as possible should be given to splitting inner loops, if it is not possi- 
85

$$
\begin{aligned}
& \text { DOSPREAD } 10 \mathrm{I}=1, \mathrm{~N} . \\
& \mathrm{A}(\mathrm{I})=\mathrm{B}(\mathrm{I})+\mathrm{C}(\mathrm{I}) S_{1} \\
& \mathrm{~B}(\mathrm{I})=\mathrm{A}(\mathrm{I}-2)+\mathrm{E}(\mathrm{I}) S_{2} \\
& \mathrm{H}(\mathrm{I})=\mathrm{P}(\mathrm{I})+\mathrm{Q}(\mathrm{I}) S_{3} \\
& \mathrm{R}(\mathrm{I})=\mathrm{H}(\mathrm{I}-4)+\mathrm{J}(\mathrm{I}) S_{4}
\end{aligned}
$$

10. CONTINUE .

(a) A Loop

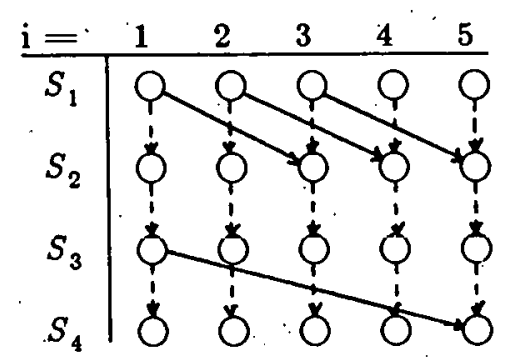

(b) The CPG for (a)

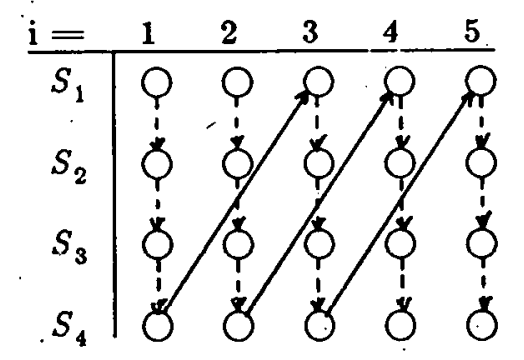

(c) The CPG of (b) after Folding

Figure 55. Folding Two LFDs into a single LBD 


\section{CHAPTER 5}

\section{CONCLUSIONS}

This chapter covers three major topics: meshing the algorithms of the last three chapters into an overall algorithm for synchronization, our conclusions about automatic synchronization, and areas for further study.

\subsection{A Global Algorithm for Synchronization}

Synchronization of programs proceeds in much the same order that the algorithms have been presented in this thesis. Each loop nest is first examined, and the most useful parallelism extracted. Once this is done, it is known which loops must be synchronized.

Next a DDG is constructed for the dospread loop. The loop is examined for exit-ifs, and if any are found the DDG is modified accordingly. Every dependence is then examined, and those that have a source or a sink in a nested loop are dealt with. This will require modifying the DDG, and if loop-splitting is performed, the source program. At this point the DDG is completed.

Redundant dependences can now be removed from the DDG. The DDG is passed on to the flow of control algorithms, which emit synchronization instructions in the proper places. . This series of steps, is then repeated for each dospread loop in the program.

These steps are summarized in Figure 56.

\subsection{Conclusions}

We have implemented many of the algorithms of this thesis in the SYNCH pass of the Parafrase compiler. The SYNCH pass runs as the last pass in the compiler; previous passes detect parallel loops and perform program transformations that aid in the detection of parallel 
Schedule loops using the algorithm of Figure 6, Chapter 1

foreach loop $L$ do

Build a DDG

$T \cdot:=\phi$

foreach statement $s$ in the loop do

if $s$ is a branch with targets $t$ outside of $L$ then add $t$ to $T$

if $T \neq \phi$ then

$/^{*}$ exit-ifs are present $* /$

perform the algorithm of Figure 16, Chapter 2

perform the algorithm of Figure 43, Chapter 3

/* take care of dependences within nested loops */

perform the algorithm of Figure 28, Chapter 2

$/^{*}$ begin dependence elimination */

/* build a CPG */

perform the algorithm of Figure 54, Chapter 4

$/^{*}$ generate instructions */

$/^{*}$ build the statement level flow of control graph */

perform the algorithm of Figure 45, Chapter 3

foreach synchronization $\alpha$ in the DDG do

$/^{*}$ add test instructions */

perform the algorithm of Figure 46, Chapter 3

$/ *$ add set or testset instructions */

perform the algorithm of Figure 47, Chapter 3

Figure 56. A Global Algorithm for Synchronization

loops. The SYNCH pass generates test and testset instructions. The algorithms implemented form a subset of the algorithms discussed in this thesis that is sufficient to insure the legal execution of a program on a multiprocessor.

The SYNCH pass does the following to add synchronization: nested loops are handled correctly, although loop splitting is not done at this time; dependence elimination is done; dependence folding is done for overlapping LFDs; and flow of control is handled. The addition of 
dependence elimination and loop splitting as discussed in this thesis is planned for the near future. Using eight synchronization registers, the need for general dependence folding has not been shown, and will probably not be implemented.

The running time of the pass is on the order of the running time needed to detect doacross loops, i.e. it is fairly long for a standard Parafrase pass, but not excessively so. As well, no effort was made in the writing of the software to optimize the running time of the pass.

Since the algorithms for generating synchronization.instructions of the types mentioned in this thesis can be made to run as a small portion of the total execution time of the entire process of automatically detecting parallelism, it is reasonable that these instructions should be generated automatically. Synchronization, like many transformation to detect and control parallelism, essentially involves the analysis of a data dependence graph. Manual data dependence analysis seems to be an operation for which humans are ill-suited. Therefore, even compilers which accept programs in parallel form should have as an option the generation of synchronization for loops marked parallel. Errors resulting from incorrect. synchronization can be difficult to detect since they are often not repeatable.

\subsection{Areas for Future Research}

Several areas for research remain. It is obvious from the algorithms of Chapter 4 that what dependences can be eliminated or folded into other dependences depends strongly on the control structure of the target architecture and the synchronization instruction set of that architecture. The synchronization overhead associated with a loop depends on the number of synchronization instructions in the loop, the execution time for each synchronization instruction to execute, and the degree of inherent parallelism allowed to remain in the loop after'synchronization. All three of these of these factors depend on the synchronization instruction set as well as on the structure of the loop's DDG. The tradeoffs between the complexity of a synchronization instruction set, the execution time of the synchronization instructions, the effects of a synchroni- 
zation instruction set on dependence elimination, and the amount of parallelism robbed from the loop by the synchronization instruction set is not well understood.

The topic of synchronizing recurrences is closely related to the amount of parallelism that is allowed to remain in a loop. It is impossible, given the synchronization instruction sets of this thesis, to adequately synchronize any recurrence. Given the more general synchronization of [ZhYe84] recurrences can be synchronized, but the overhead associated with synchronizing a general recurrence is, enormous and overwhelms any savings in time gained by running the recurrence in parallel. It is desirable that strategies be developed to recognize special dependence patterns and that optimal synchronization strategies be developed for them.

Finally, the generation of synchronization instructions for multiply nested loops has only been barely touched on in this thesis. Although dependence elimination will not be much more difficult than for a single level of spread parallelism, the issues surrounding the placement, generation and types of synchronization instructions for multiple levels of spread parallelism are not so clear. 


\section{REFERENCES}

[AhU177] : A.V. Aho and J.D. Ullman, Principles of Compiler Design, Addison-Wesley, Reading, Massachusetts, 1977.

[Alli85] Alliant Computer Systems Corp., "FX/Series Architecture Manual," Acton, Massacliuselts, 1985

[Bane79] U. Banerjee, "Speedup of Ordinary Programs," Ph.D. Thesis, University of Illinois at Urbana-Champaign, DCS Report No. UIUCDCS-R-79-989, October 1979.

[Chen83] S. Chen, "Large-scale and High-speed Multiprocessor System for Scientific Applications = Cray-X-MP-2 Series," Proc. of NATO Advanced Rescarch Workshop on High Speed Computing, Kawalik(Editor), pp. 59-67, June 1983.

[Cytr84] R.G. Cytron, "Compile-time Scheduling and Optimization for Asynchronous Machines," Ph.D. Thesis, University of Illinois at Urbana Champaign, DCS Report No. UIUCDCS-R-84-1177, 1984.

[Davi81] J.R. Beckman-Davies, "Parallel Loop Constructs for Multiprocessors," 'M.S. Thesis, University of Illinois at Urbana-Champaign, DCS Report No. UIUCDCSR-81-1070, Mny 1981.

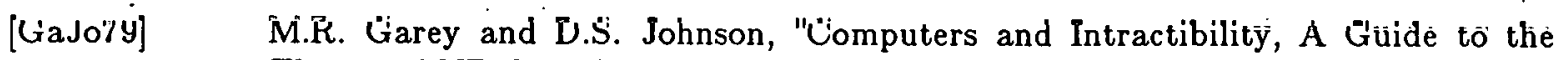
Theory of NP-Completeness," W.H. Freeman and Company, San Francisco, California, 1979.

[GGKM83] A. Gottlieb, R. Grishman, C.P. Kruskal, K.P. McÁliffe, L. Rudolph, and M.

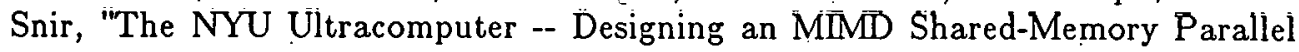
Machino;" IEEE Trano. on Computoro, Vol. C 33, No. 3, pp. 175-180, Fobruary 1083.

[GKLS83] D. Gajoki, D. Kuok, D. Lawrio and A. Samoh, "GEDAR - A Largo Scalo Multiprocessor," Proc. of International Conference on Parallel Processing, pp. 524529, August 1983.

[LuBa80] S.F. Lundstron and G.H. Barnes, "Controllable MMD Architecture," Proceedings of the 1980 Conference on Parallel Processing, pp: 19-27, 1980.

[Kuck78] D.J. Kuck, The Structure of Computers and Computations, Volume 1, John Wiley and Sons, New York, 1978. 
[KKLW80] D.J. Kuck, R.H. Kuhn, B. Leasure, and M. Wolfe, "The Structure of an Advanced Vectorizer for Pipelined Processors," Fourth International Computer Software and Applications Conference, October, 1980.

[KLPL81] - D.J. Kuck, R. Kuhn, D. Padua, B. Leasure, and M. Wolfe, "Depedence Graphs and Compiler Optimizations," Proceedings of the 8-th ACM Symposium on Principles of Programming Languages, pp. 207-218, January 1981.

[LiZh85] Zhiyuan Li, "A Technique for Reducing Data Synchronization in Multiprocessed Loops," MS. Thesis, University of Illinois at Urbana-Champaign, CSRD Report No. 521, 1985.

[Padu79] D.A. Padua Haiek, "Multiprocessors: Discussions of Some Theoretical and Practical Problems," Ph.D. Thesis, University of Illinois at Urbana-Champaign, DCS Report No. UIUCDCS-R-79-990, November 1979.

[Poly86]. C.D. Polychronopolous, Ph.D. Thesis, Work in Progress, University of Illinois at Urbana-Champaign, 1986.

[Smit81] B. Smith, "Architecture and Applications of the HEP Multiprocessor Computer System," Real Time Procssing IV, Proc. of SPIE, pp. 241-248, 1981.

[Veid85] A. Veidenbaum, "Compiler Optimizations and Architecture Design Issues for Mulitprocessors," Ph.D. Thesis, University of Illinois at Urbana-Champaign, CSRD Report. No. 520, 1985.

[ZhYe84] C.Q. Zhu and P.C. Yew, "A Synchronization Scheme and Its Applications for Large Multiprocessor Systems," Proc. of the 1984 International Conference on Distributed Computing Systems, pp. 486-493, May 1984.

[Wolf82] M.J. Wolfe, "Optimizing Supercompilers for Supercomputers," Ph.D. Thesis, University of Illinois at Urbana-Champaign, DCS Report No. UIUCDCS-R-821105, 1982 . 


\begin{tabular}{|c|c|c|c|}
\hline $\begin{array}{l}\text { BIBLIOGRAPHIC DATA } \\
\text { SHEET }\end{array}$ & $\begin{array}{ll}\text { 1. Report No. } & \\
\text { CSRD }-588 \\
\end{array}$ & 2. & 3. Recipient's Accession No. \\
\hline \multirow{2}{*}{\multicolumn{2}{|c|}{$\begin{array}{l}\text { 4. Title and Subtitle } \\
\text { AUTOMATIC GENERATION OF SYNCHRONIZATION } \\
\text { INSTRUCTIONS FOR PARALLEL PROCESSORS }\end{array}$}} & & $\begin{array}{l}\text { 5. Report Date } \\
\text { May } 1986\end{array}$ \\
\hline & & & 6. \\
\hline \multicolumn{3}{|l|}{ 7. Author(s) } & $\begin{array}{l}\text { 8. Performing Organization Rept. } \\
\text { No. }\end{array}$ \\
\hline \multirow{2}{*}{\multicolumn{3}{|c|}{$\begin{array}{l}\text { 9. Performing Organization Name and Address } \\
\text { University of I11inois at Urbana-Champaign } \\
\text { Center for Supercomputing Research and Development } \\
\text { Urbana, IL } 61801-2932\end{array}$}} & 10. Project/Task/Work Un it No. \\
\hline & & & $\begin{array}{l}\text { 11. Contract/Grant No.US NSF D } \\
101.10 ; \text { US NSF DCR84-069 } \\
\text { US DOE-DE-FG02-85ER2500 }\end{array}$ \\
\hline \multirow{2}{*}{\multicolumn{3}{|c|}{$\begin{array}{l}\text { 12. Sponsoring Organization Name and Address } \\
\text { Nationa1 Science Foundation, Washington, DC; and } \\
\text { U.S. Department of Energy, Washington; DC }\end{array}$}} & $\begin{array}{l}\text { 13. Type of Report \& Period } \\
\text { Covered } \\
\text { Master's Thesis }\end{array}$ \\
\hline & & & 14. \\
\hline
\end{tabular}

16. Abstracts

The development of high speed parallel multi-processors, capable of parallel execution of doacross and forall loops, has stimulated the development of compilers to transform serial FORTRAN programs to parallel forms. One of the duties of such a compiler must be to place synchronization instructions in the parallel version of the program to insure the legal execution order of doacross and foral1 1 oops.

This thesis gives strategies usable by a compiler to generate these synchronization instructions. It presents algorithms for reducing the parallelism in FORTRAN programs to match a target architecture, recovering some of the parallelism so discarded, and reducing the number of synchronization instructions that must be added to a FORTRAN program, as we11 as basic. strategies for placing synchronization instructions. These algorithms are developed for two synchronization instruction sets.

17. Key Words and Document Analysis. 17a. Descriptors

compilers

languages

software

synchronization

17b. Identifiers/Open-Ended Terms

17c. COSATI Field/Group

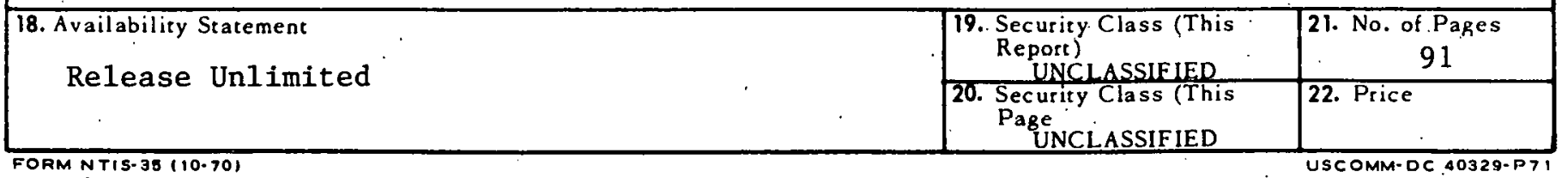

